\title{
Low PAPR reference signal transceiver design for 3GPP 5G NR uplink
}

\author{
M Sibgath Ali Khan* (D), Koteswara Rao, Saidhiraj Amuru and Kiran Kuchi
}

*Correspondence: ee13p0003@iith.ac.in

Department of Electrical Engineering, Indian Institute of

Technology - Hyderabad,

Telangana, India

\section{Springer Open}

\begin{abstract}
Low peak-to-average-power ratio (PAPR) transmissions significantly improve the cell coverage as they enable high power transmissions without saturating the power amplifier. To support the low PAPR transmissions, $\pi / 2$-BPSK-based data and DMRS are introduced in the latest version of $5 \mathrm{G}$ NR specifications. In addition to that, the spatial multiplexing support is also extended to $\pi / 2$-BPSK data transmissions. The DMRS sequences corresponding to these spatial streams (users) are frequency division multiplexed (FDM). However, the spectrum shaping process employed in the generation of $\pi / 2$-BPSK waveforms is frequency selective and hence results in asymmetric spectrum shaping effect on DMRS sequences, when they are frequency multiplexed. This subsequently results in a non-uniform block error (BLER) and PAPR performances across the spatial users, which in turn may result in inter user interference across the spatial users. In this paper, we propose two transmitter architectures, namely method 1 and method 2, to generate low PAPR $\pi / 2$-BPSK-based DMRS waveforms. The proposed architectures ensure that the spectrum shaping effect is uniform across all the spatial streams. We corroborate through simulations that the proposed architectures will result in identical block error and PAPR performances across all the spatial streams.
\end{abstract}

Keywords: PAPR, Spectrum shaping filter, Impulse response, BPSK

\section{Introduction}

For a cellular network, uplink transmissions define the coverage area. The transmission power in the uplink is limited to $23 \mathrm{dBm}$ as opposed to $43 \mathrm{dBm}$ in the downlink [1], owing to hardware limitations (such a battery size) and regulatory constraints. This limited transmission power in the uplink must, therefore, be judiciously used to enhance the cell coverage without increasing the CAPEX/OPEX costs of deploying more cell sites. Therefore the uplink design of a cellular standard is often crucial.

To enhance the cell coverage of the newly designed 3GPP 5G NR, a new modulation scheme, namely $\pi / 2$-BPSK, was introduced for the uplink data channel (physical uplink shared channel- PUSCH) and control channel (physical uplink control channel - PUCCH) transmissions. The $\pi / 2$-BPSK modulation scheme, when transmitted using discrete Fourier transform spread orthogonal frequency division multiplexing (DFT-sOFDM waveform), offers low PAPR when compared to higher-order modulation schemes

(c) The Author(s). 2020 Open Access This article is licensed under a Creative Commons Attribution 4.0 International License, which permits use, sharing, adaptation, distribution and reproduction in any medium or format, as long as you give appropriate credit to the original author(s) and the source, provide a link to the Creative Commons licence, and indicate if changes were made. The images or other third party material in this article are included in the article's Creative Commons licence, unless indicated otherwise in a credit line to the material. If material is not included in the article's Creative Commons licence and your intended use is not permitted by statutory regulation or exceeds the permitted use, you will need to obtain permission directly from the copyright holder. To view a copy of this licence, visit http://creativecommons.org/licenses/by/4.0/. 
including QPSK, 16-QAM, and others. The complementary CDF (CCDF) of PAPR for various modulation schemes is shown in Fig. 1, which clearly shows the low PAPR behavior of the $\pi / 2$-BPSK modulation scheme. To further reduce the PAPR, spectrum shaping is applied to the $\pi / 2$-BPSK symbols. Spectrum shaping is a PAPR reduction technique which can be performed either in time domain or frequency domain [2,3]. Specifically, the PAPR of the $\pi / 2$-BPSK modulation scheme with DFT-s-OFDM waveform and spectrum shaping is smaller than $2 \mathrm{~dB}$. Moreover, it is shown in $[2,3]$ that the power amplifier can be driven to saturation, and yet the error rate performance of this modulation scheme is not compromised. Hence, this modulation scheme plays a crucial role in significantly enhancing the cell coverage for 3GPP 5G NR-based cellular networks.

The demodulation reference signals (DMRS) employed for coherent data demodulation in uplink of the current 5G NR systems are generated using Zadoff-Chu (ZC) sequences or QPSK-based computer-generated sequences(CGS) as specified in Section 5.2.2 in [4] and Section 6.2.2 in [5]. The CCDF of PAPR for a DFT-s-OFDM waveform with spectrum-shaped $\pi / 2$-BPSK data symbols and the Zadoff-Chu based DMRS sequences is shown in Fig. 2. It can be seen that PAPR of $\pi / 2$-BPSK data is lower than that of the $\mathrm{ZC}$ sequences by over $2 \mathrm{~dB}$. Therefore, even though the data transmissions have low PAPR and potentially allow for larger coverage, the DMRS design still limits the cell size due to its high PAPR. For this reason, 3GPP introduced a new study item in Rel-16 to design new reference sequences with lower PAPR [6]. In one of our associated contribution [7], a step-by-step procedure to generate low PAPR DMRS sequences for various lengths is provided. The sequences in $[8,10-13]$ were agreed to be used as low-PAPR reference sequences. In this paper, we employ them as input to the proposed transceiver architectures.

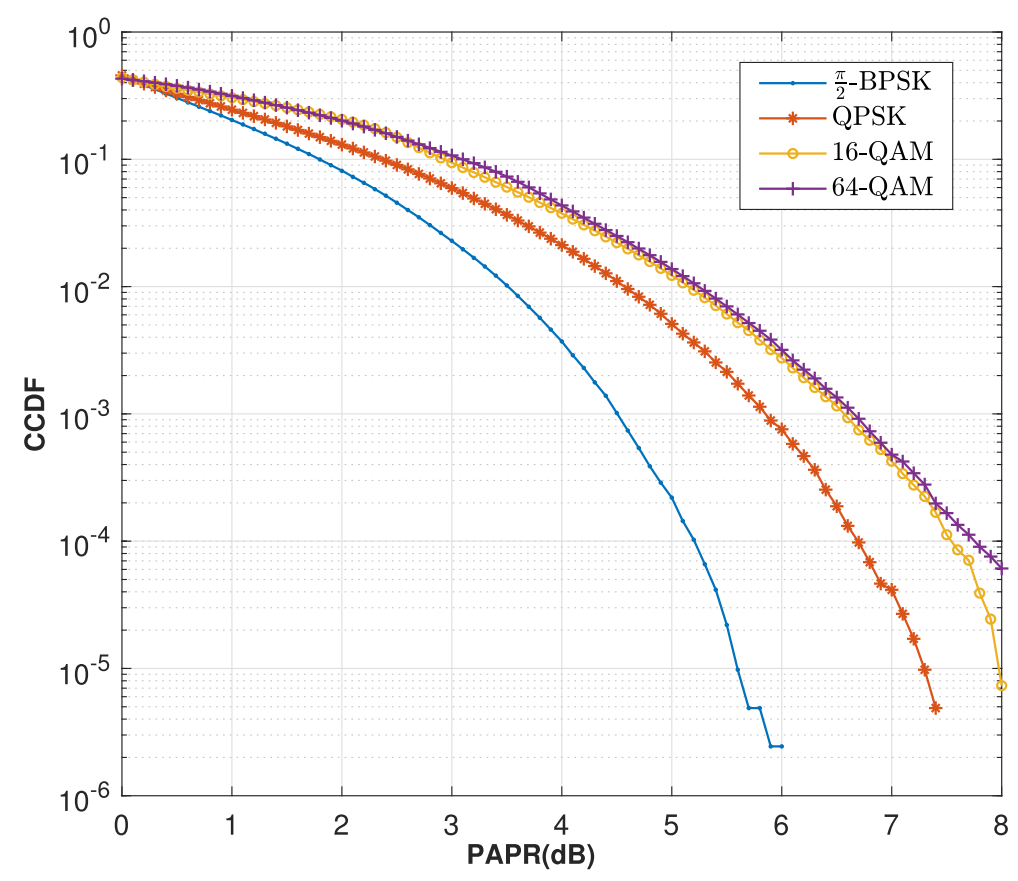

Fig. 1 PAPR of different modulation schemes using a DFT-s-OFDM waveform 


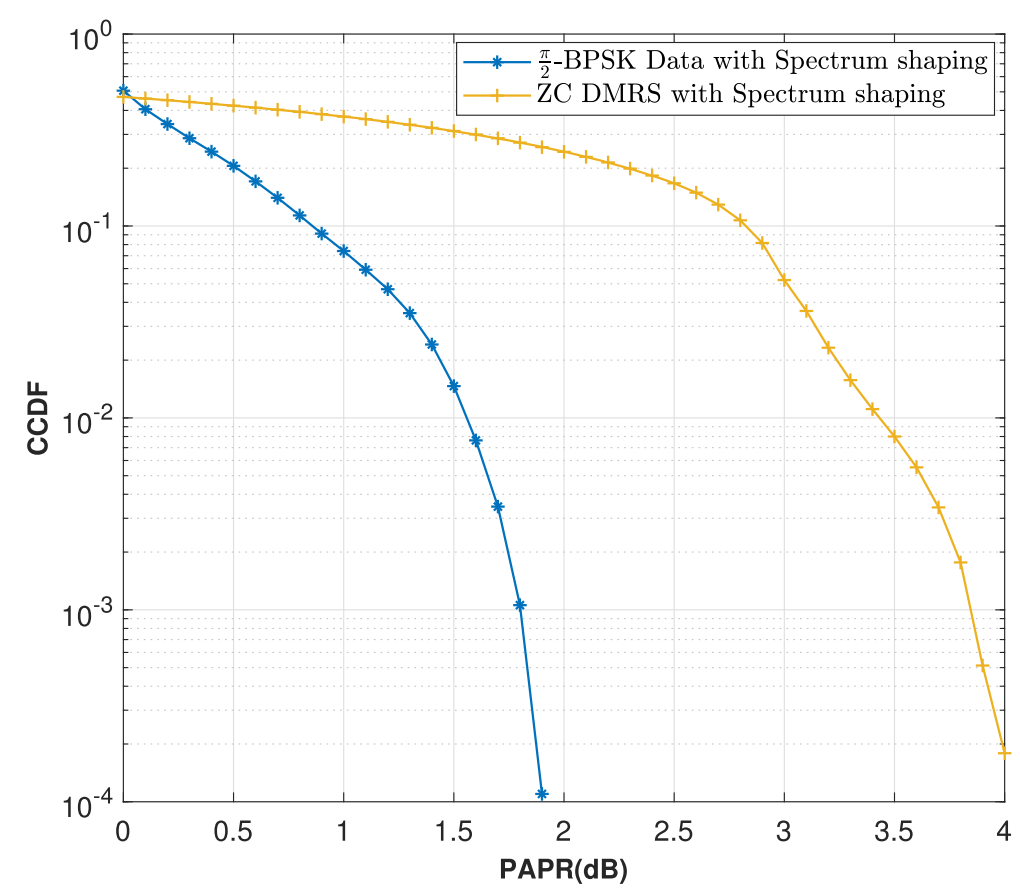

Fig. 2 PAPR comparison between spectrum-shaped ZC sequence and spectrum-shaped $\pi / 2$-BPSK data

The specifications for 5G NR also support multiple stream transmissions using the DFT-s-OFDM waveform. In other words, a single user can be scheduled to transmit multiple streams, or multiple users can be configured simultaneously to transmit multiple streams depending on the channel conditions. In order to support these multiple-stream transmissions, multiple DMRS sequences are required, one for each stream. This is achieved by introducing the concept of baseband DMRS port, where one single port is assigned for demodulation of each stream [4, Sec 6.3.1.3]. Where in [4, Sec 4.4.1], a DMRS port is defined such that the channel over which a symbol on the DMRS port is conveyed can be inferred from the channel over which another symbol on the same DMRS port is conveyed. Since the DMRS of each stream must be independently decoded to derive channel estimates, the DMRS sequences must be orthogonally separated to avoid any interference.

The orthogonality across the ports can be achieved by either frequency division multiplexing $(\mathrm{FDM})$ or code division multiplexing $(\mathrm{CDM})$ or time-division multiplexing (TDM). However, the current 3GPP 5G NR [8] supports only FDM with a maximum of two streams when DFT-s-OFDM waveform is enabled. In the FDM method, the same DMRS sequence is employed by all the DMRS ports, but orthogonalized in frequency, as shown in Fig. 3, in FDM, the length of DMRS on each port will be $\frac{M}{Q}$, corresponding to the data allocation of length $M$ subcarriers, where $Q$ indicates the number of DMRS ports, which is limited to 2 (port -0 and port -1 ) as per the current 3GPP specifications [9] and also for the scope of this paper. A typical data DMRS multiplexing in the current 5G NR systems is shown in Fig. 4. Here, a few of the DFT-s-OFDM symbols are dedicated for DMRS transmission while the other symbols carry the user data. Channel estimates derived from these DMRS symbols will be employed for coherent demodulation of data. 


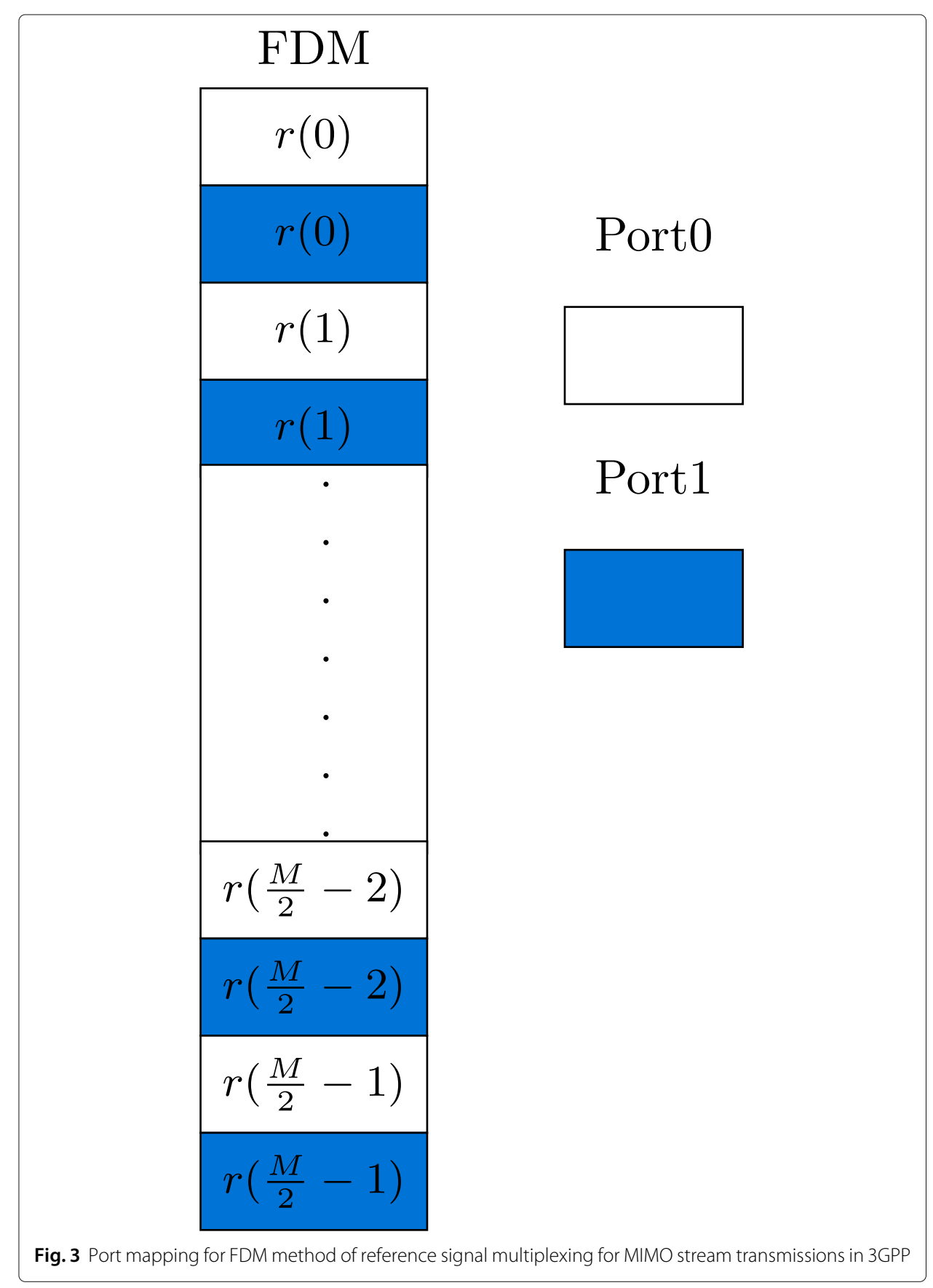

The existing ZC or QPSK based DMRS generation does not include the spectrum shaping; however, spectrum shaping for $\pi / 2$-BPSK data waveform is briefly outlined in [1]. To accurately derive the channel estimates from DMRS for coherent demodulation, the spectrum shaping has to align between data and DMRS transmissions. Since the exact spectrum shaping procedure for DMRS is not known, and if we continue to follow the same spectrum shaping principles for DMRS as well as data, then it results in asymmetric spectrum shaping effect on port-0 and port-1, which eventually result in port-specific channel estimation and PAPR performances, which will be shown later in the Section 4. 


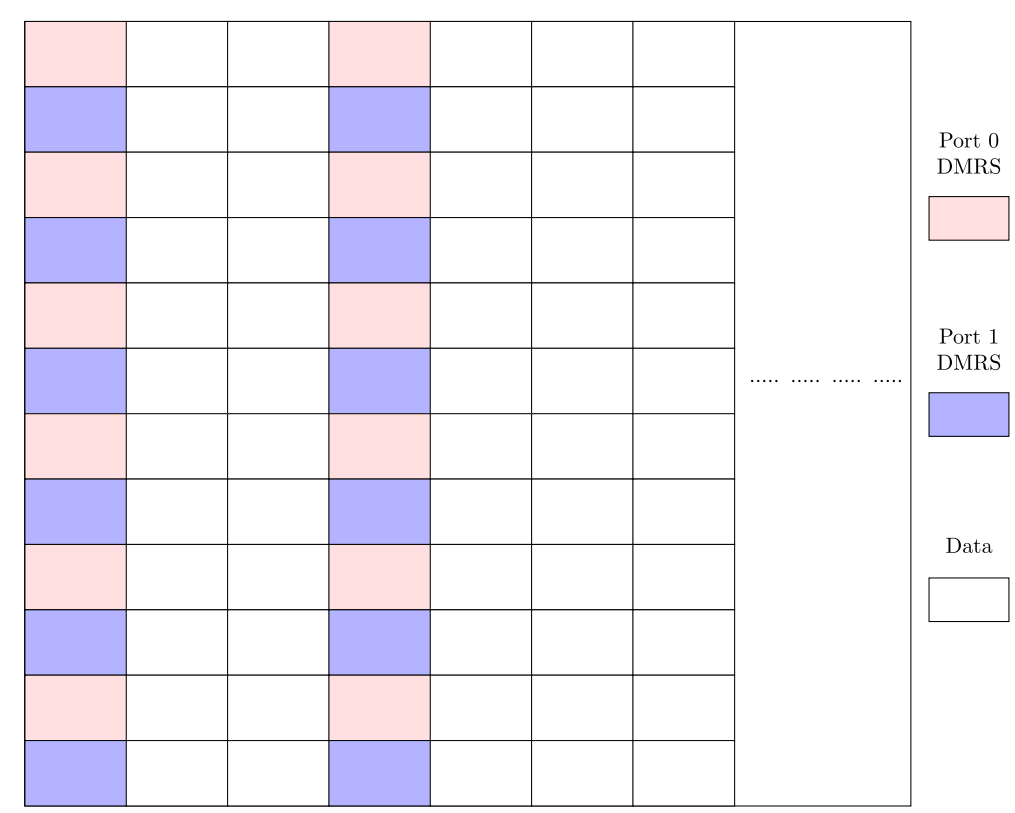

Fig. 4 DMRS and data symbols in a OFDM resource grid

In this work, we address the problem of Low PAPR DMRS waveform generation in the context of spatial multiplexing. The key contributions in the paper are

1 Firstly, we present the generation of $\pi / 2$-BPSK based DMRS waveform and show that the PAPR of $\pi / 2$-BPSK based DMRS waveforms is almost $2 \mathrm{~dB}$ lesser than the conventional ZC based DMRS waveform.

2 For the case of spatial multiplexing, we propose two transmitter architectures, namely transmission method 1 and transmission method 2 . The proposed methods ensure that the spectrum shaping is aligned across all the scheduled DMRS ports and thereby similar PAPR and BLER performances across the ports.

3 Transmission method 1 implements the spectrum shaping in frequency domain, where some fundamental DFT properties are invoked to ensure the similar spectrum shaping effect.

4. Transmission method 2 implements the spectrum shaping in the time domain through convolution procedure.

5 We establish the equivalence between method 1 and method 2 and show that the DMRS waveforms generated by both the methods are exactly identical.

At the receiver, we employ a DFT-based channel estimation procedure to estimate the joint impulse response of the wireless channel and spectrum shaping filter, which further is employed for data demodulation. We corroborate through simulations that both transmission method 1 and transmission method 2 result in identical channel estimation and PAPR performance on all the scheduled DMRS ports. Note that, as per the latest $5 \mathrm{G}$ specifications [9], when $\pi / 2$-BPSK data transmission is enabled, a maximum of 2 spatial users are supported. Hence, the analysis in this paper is confined to 2 DMRS ports; however, the proposed methods as such can be readily extended to any number of DMRS ports, which is explained later in Sections 2.4 and 2.5. 
The rest of the paper is organized as follows. The $\pi / 2$-BPSK data and $\pi / 2$-BPSK DMRS generation using the proposed transmitter designs is discussed in Section 2 . The receiver architecture, mainly the channel estimator employed to derive the channel estimates on both port- 0 and port-1, is described in Section 3. The block error rate(BLER) and PAPR performances comparing the proposed and existing designs are presented and discussed under Section 4. Finally, we summarize and conclude the paper in Section 5.

Notation: The following notations are used in this paper. Bold uppercase letters $\mathbf{X}$ denote matrices, bold lowercase letters $\mathbf{x}$ denote vectors, non-boldface letters represent scalars, and $\mathbf{x}_{t}, \mathbf{y}_{f}$ indicates the time domain and frequency domain vectors $x$ and $y$ respectively. $\mathbf{x}^{T}$ and $\mathbf{X}^{\dagger}$ represent the transpose and Hermitian operations on the vector $\mathbf{x}$ and matrix $\mathbf{X}$ respectively. We use the symbol $\mathbf{x}$ to denote the data symbols and $\mathbf{r}$ to denote reference signal symbols. $\mathbf{x} . \mathbf{y}$ represents the Hadamard product of vectors $\mathbf{x}$ and $\mathbf{y}$.

\section{Transmitter architecture for $\pi$ / 2-BPSK data and DMRS generation}

In this section, we present transmitter designs to generate low PAPR data and DMRS waveforms. We first describe the system model, including the design of the DFT-s-OFDM data waveform as per the current 5G NR specifications, and then discuss the proposed transmitter designs.

\section{$2.1 \pi / 2$-BPSK symbol generation}

The current NR specifications $[4,5]$ specify DFT-s-OFDM for uplink transmissions in the coverage limited scenarios. Also, in addition to QAM modulation techniques, a new modulation scheme, namely, $\pi / 2$-BPSK, was introduced in 5G NR. In BPSK modulation; the input bit sequence $b(m)$ is mapped to complex-valued modulation symbol $x_{t}(m)$ as given below

$$
x_{t}(m)=\frac{1}{\sqrt{2}}[1-2 b(m)+i(1-2 b(m))] .
$$

whereas $\pi / 2$-BPSK is a special constellation-rotated BPSK modulation, in which the evennumbered symbols are transmitted as like in BPSK, and the odd-numbered symbols are phase rotated by $\pi / 2$ as given in (1)

$$
x_{p}(m)=e^{i(m \bmod 2) \frac{\pi}{2}} x_{t}(m), m \in[0, \ldots, M-1] .
$$

Here, the subscript $p$ in $x_{p}(m)$ indicates a phase rotated sequence and the sub-script $t$ in $x_{t}(m)$ indicates a time domain sequence. $i=\sqrt{-1}$ and $M$ is the length of a BPSK sequence $x_{t}(m)$.

Note: In order to maintain consistency with the other modulation mappings, for BPSK, the input bits are mapped to the rotated PSK [4], rather than from the set $\{-1,1\}$,

The $\pi / 2$-phase rotation can be equivalently expressed in vector notation as given below,

$$
\mathbf{x}_{p}=\frac{1+i}{\sqrt{2}} \mathbf{P} \mathbf{x}_{t}
$$

where $\mathbf{x}_{t}$ is a $M$ length BPSK vector and $\mathbf{P}$ is $M \times M$ diagonal matrix with diagonal entries $p_{m m}=e^{i(\bmod 2) \frac{\pi}{2}}$. Note that, although the constellation is similar to QPSK, we can only transmit 1-bit on one $\pi / 2$-BPSK modulation symbol. 


\subsection{Spectrum shaping}

The $\pi / 2$-BPSK symbols, when combined with an appropriate spectrum shaping, enables low PAPR transmissions without compromising the error rate performance [2-4]. Spectrum shaping can be performed either in time or frequency domain. In the case of frequency-domain processing, spectrum shaping can be performed by means of a spectrum-shaping function $\mathbf{w}_{f}=\mathbf{D}_{M} \mathbf{w}_{t}$, where $\mathbf{w}_{t}$ is zero-padded time domain impulse response of the $L$-tap spectrum shaping filter i.e., $\mathbf{w}_{t}=$

$$
\begin{gathered}
{[w(0), w(1), . . w(L-1), \underbrace{0, \ldots, 0}_{M-L}]^{T} \text {. Where } \mathbf{D}_{M} \text { is an } M \times M \text { DFT matrix given by }} \\
\mathbf{D}_{M}(k, m)=e^{\frac{-i 2 \pi k m}{M}}, 0 \leq k, m \leq M-1 .
\end{gathered}
$$

Remark on the length of the spectrum shaping filter: The spectrum shaping is implementation-specific and is generally unknown at the receiver. In such a case, the receiver needs to estimate the impulse response of the spectrum shaping filter and wireless channel jointly. Let $L_{D M R S}, L_{C I R}, L_{F i l}$ be the length of the DMRS sequence, channel impulse response (CIR) of the wireless channel, and length of spectrum shaping filter, respectively. According to [12], the DMRS sequence length should be at least equal to the length of the joint impulse response of the wireless channel and the spectrum shaping filter, i.e.,

$$
L_{D M R S} \geq L_{C I R}+L_{F i l}
$$

As per $[4,5]$, the minimum allocation size for data transmissions is 12 subcarriers, for which the length of the DMRS sequence $\left(L_{D M R S}\right)$ will be 6 subcarriers for the case of twostream transmission. Also, as per 3GPP channel models [14], for an allocation size of 12 subcarriers, the maximum length of channel impulse response $\left(L_{C I R}\right)$ will be $\leq 3$. Invoking the relation in (4), it can be inferred that the spectrum shaping filter can have a maximum of 3 taps. Hence, the spectrum shaping filters should be chosen such that their impulse response is limited to 3 taps or less. Commonly employed spectrum shaping filters with 2 and 3-tap impulse response are $w_{1}=[1,-1]$ and $w_{2}=[-0.28,0,-0.28]$ respectively. The corresponding frequency spectrum of these filters is shown in Fig. 5.

\subsection{DMRS signal structure}

As discussed in Section 1, when multiple DMRS sequences are transmitted on frequency division multiplexed DMRS ports, spectrum shaping needs to be performed properly to ensure identical spectrum shaping effect on data and DMRS, which otherwise results in non-identical channel estimation performance (and thereby equalization and demodulation) across the DMRS ports, which is not desirable.

Hence, the DMRS transmitter design, besides minimizing the PAPR of the waveform, should also ensure that the characteristics of the waveform (like auto-correlation and cross-correlation) are similar across all the DMRS ports.

\subsection{Transmission method 1}

In this section, we present method 1 of data and DMRS waveform generation, wherein the spectrum shaping is performed in the frequency domain. 


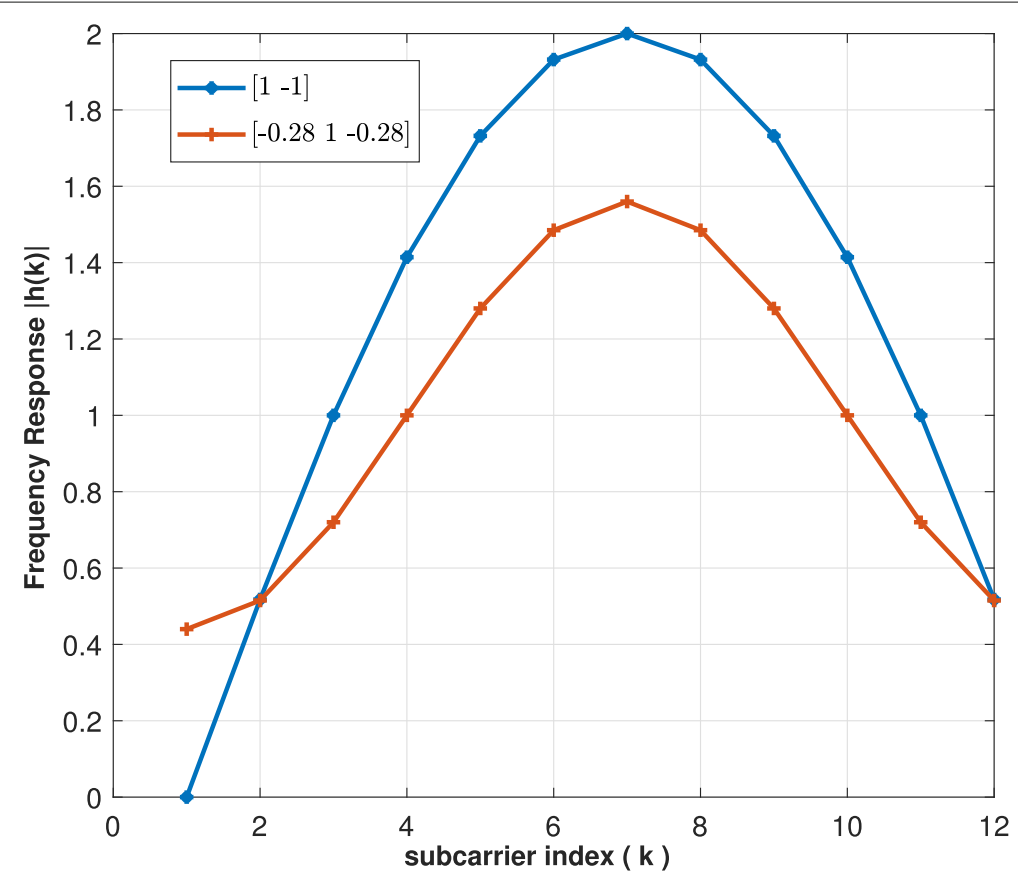

Fig. 5 Frequency response of commonly used spectrum shaping filters with 2-tap and 3-tap impulse response

\subsubsection{Data waveform design method 1}

The transmitter architecture for data waveform generation is shown in Fig. 6. Let $\mathbf{x}_{p}$ denote an $M \times 1$ vector of $\pi / 2$-BPSK modulated data symbols generated as per (1). For transmission via DFT-s-OFDM, the $\pi / 2$-BPSK data symbols are first DFT-precoded as

$$
x_{f}(k)=\sum_{m=0}^{M-1} x_{p}(m) e^{\frac{-i 2 \pi k m}{M}} .
$$

The subscript $f$ in $x_{f}(k)$ indicates a frequency domain sequence. The DFT precoding shown in (5) can be equivalently represented in vector notation form as

$$
\mathbf{x}_{f}=\mathbf{D}_{M} \mathbf{x}_{p}
$$

where $\mathbf{D}_{M}$ is a $M \times M$ DFT matrix given by (3). The spectrum shaping is performed on the DFT-precoded data vector as $\mathbf{x}_{f}^{s}=\mathbf{w}_{f} \cdot \mathbf{x}_{f}$, where $\mathbf{x}_{f}^{s}$ indicates the spectrum-shaped frequency domain data $\mathbf{x}_{f}$. The spectrum-shaped data vector $\mathbf{x}_{f}^{s}$ is then mapped to a set of subcarriers in frequency domain via an $N \times M$ mapping matrix $\mathbf{M}_{f}$ where $M \leq N$. The block diagonal mapping matrix $\mathbf{M}_{f}$ is defined below (without loss of generality, the DFT precoded data is mapped to the initial $\mathrm{M}$ subcarriers)

$$
\mathbf{M}_{f}=\left[\mathbf{I}_{M} \mathbf{0}_{N-M}\right]^{T} .
$$

Finally, the output of this mapping operation is converted to $N \times 1$ time domain vector $\mathbf{s}_{t}$ as

$$
\mathbf{s}_{t}=\mathbf{D}_{N}^{\dagger} \mathbf{M}_{f} \mathbf{x}_{f}^{s}
$$




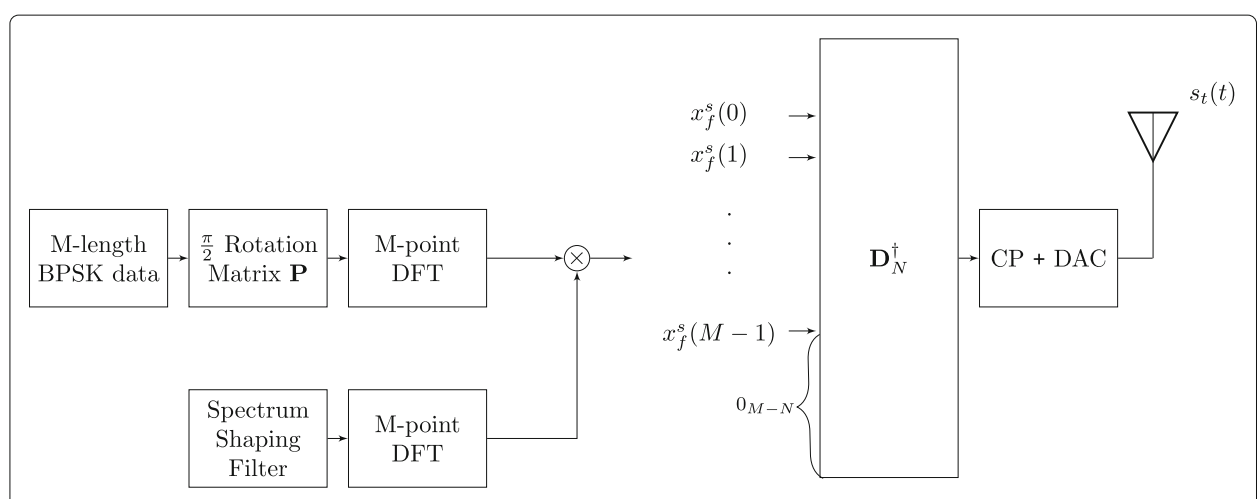

Fig. 6 Transmitter architecture for data waveform generation using method 1

where $\mathbf{D}_{N}^{\dagger}$ is an inverse DFT matrix and $N$ is the total number subcarriers corresponding to the system bandwidth. An appropriate length cyclic prefix is added to $\mathbf{s}_{t}$ to generate $\mathbf{s}_{t}(t)$ as given by equation (5.3.1) in [4].

\subsubsection{DMRS waveform design method 1}

As mentioned in Section 1, the current 5G system supports only 2 MIMO streams in the uplink, and the corresponding DMRS is multiplexed in FDM manner [10]. Hence, $\frac{M}{2}$ length DMRS sequences will be transmitted on port 0 and port 1 corresponding to $M$ length data allocation. We will next present transmitter architectures for port 0 and port 1 , respectively, such that the resultant DMRS waveforms from either of the ports have low PAPR and similar characteristics.

The transmitter architectures for port 0 and port 1 are shown in Figs. 7 and 8. In this architecture, the transmitter design is such that a given time domain DMRS sequence $\mathbf{r}_{t}$ will result in an identical spectrum-shaped frequency domain sequence for both DMRS ports. This subsequently results in similar auto and cross-correlation properties and hence produces an identical channel estimation performance at the receiver. The summary is tabulated in Table 1.

DMRS waveform generation for port 0: Let $\mathbf{r}_{t}$ be a pre-determined $\frac{M}{2}$ length DMRS sequence with BPSK modulated symbols chosen as per the designs in [6-13]. This will be

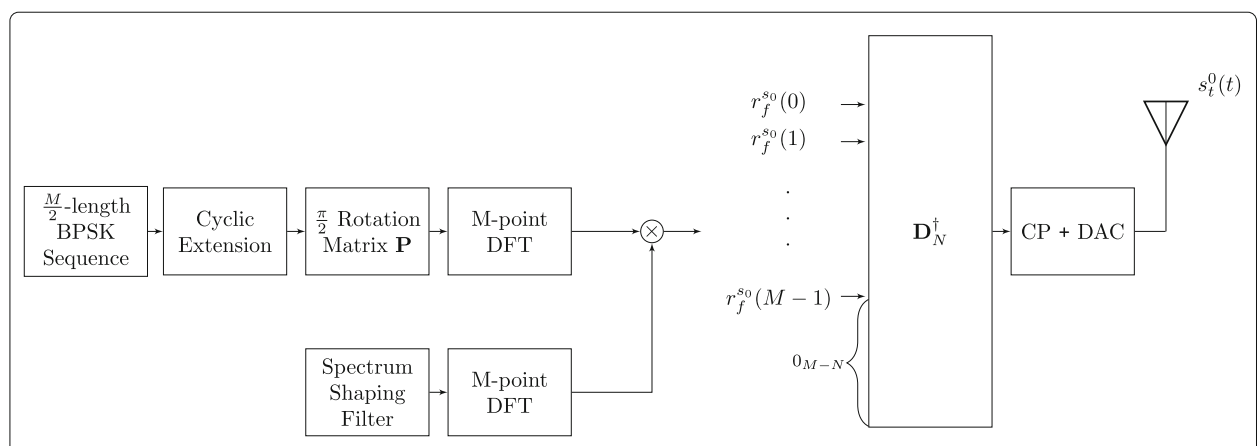

Fig. 7 Transmitter architecture for port 0 DMRS waveform generation using method 1 


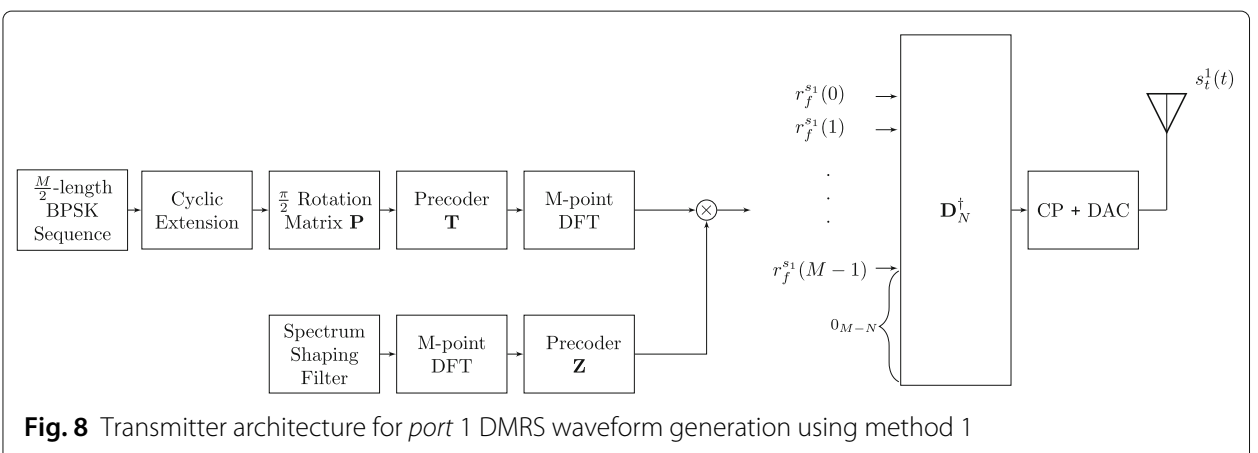

cyclically extended to give a $M$ length vector $\tilde{r}_{t}(n)$ as follows

$$
\tilde{r}_{t}(n)=r_{t}\left(n \bmod \frac{M}{2}\right), n=0,1, \ldots, M-1 .
$$

Using the diagonal matrix $\mathbf{P}$ defined in (2), a $\pi / 2$-phase rotation is applied on $\tilde{r}_{t}$ to give $\tilde{r}_{t}^{p}=\mathbf{P} \tilde{r}_{t}$. The resultant $\pi / 2$-BPSK sequence is DFT precoded as $\mathbf{r}_{f}^{p_{0}}=\mathbf{D}_{M} \tilde{r}_{t}^{p}$. Invoking the DFT property that when a sequence is repeated twice in time domain, then it will have a comb like structure in frequency domain. Hence, the DFT output $\mathbf{r}_{f}^{p_{0}}$ will have non-zero entries at the even locations as shown in Fig. 3 (and hence the notation $\mathbf{r}_{f}^{p_{0}}$ ). The DFTprecoded DMRS symbols are spectrum-shaped using $\mathbf{w}_{f}$ defined in Section 2.2 to give the spectrum-shaped port-0 DMRS as

$$
\mathbf{r}_{f}^{s_{0}}=\mathbf{w}_{f} \cdot \mathbf{r}_{f}^{p_{0}} .
$$

DMRS waveform generation for port 1: When multiple DMRS ports are frequency multiplexed, the DMRS sequence should be identical on both ports [8] i.e., the input BPSK sequence $\mathbf{r}_{t}$ and the resulting $\frac{\pi}{2}$ BPSK sequence $\tilde{r}_{t}^{p}$ has to be same for both port 0 and port 1. However, different from port 0 , to generate the spectrum-shaped frequency domainDMRS sequence on port 1 , the following additional steps need to be performed

- a precoder $\mathbf{T}$ is applied on $\tilde{r}_{t}^{p}$, where $\mathbf{T}$ is a $M \times M$-diagonal matrix with diagonal entries $T_{m m}=e^{i 2 \pi m / M}$ followed by DFT precoding as shown below

$$
\mathbf{r}_{f}^{p_{1}}=\mathbf{D}_{M} \mathbf{T} \tilde{r}_{t}^{p} .
$$

Invoking the DFT property defined in the Section 2.4.2 and the frequency shift property, it can be seen that $\mathbf{r}_{f}^{p_{1}}$ is a comb-like structure with non-zero entries only at odd subcarriers equivalent to port-1 mapping as given in Fig. 3.

- Spectrum shaping of $\mathbf{r}_{f}^{p_{1}}$ is done as follows,

$$
\mathbf{r}_{f}^{s_{1}}=\left(\mathbf{Z} \mathbf{w}_{f}\right) \cdot \mathbf{r}_{f}^{p_{1}}
$$

Table 1 Summary of method 1 based DMRS waveform generation

\begin{tabular}{llll}
\hline Port & Time domain DMRS & Spectrum shaping filter & Freq domain DMRS \\
\hline 0 & $\tilde{r}_{t}^{D}(n)=\mathbf{P r}_{t}\left(n \bmod \frac{M}{2}\right)$ & $\mathbf{w}_{f}=\mathbf{D}_{M} \mathbf{w}_{t}$ & $\mathbf{D}_{M} \tilde{r}_{t}^{p} \cdot \mathbf{w}_{f}$ \\
1 & $\tilde{r}_{t}^{p}(n)=\mathbf{T P r}_{t}\left(n \bmod \frac{M}{2}\right)$ & $\mathbf{w}_{f}=\mathbf{Z} \mathbf{D}_{M} \mathbf{w}_{t}$ & $\mathbf{D}_{M} \tilde{r}_{t}^{p} \cdot \mathbf{w}_{f}$ \\
\hline
\end{tabular}


where, $\mathbf{Z}$ is a square-circulant matrix of size $M \times M$ whose 1 st row entries are

$$
[\underbrace{0,0, \ldots, 0}_{M-1}, 1] \text {. }
$$

Note: The precoder $(\mathbf{Z})$ on the spectrum shaping filter $\left(w_{f}\right)$ circularly rotate the frequency coefficients of spectrum shaping filter by 1 sample for port 1 . This ensures that the same set of filter coefficients gets applied on $\pi / 2$-BPSK sequence $\tilde{r}_{t}^{p}$ for both port 0 and port 1 DMRS. Hence, the effect of spectrum shaping is identical on both port 0 and port 1 DMRS, whereas, without precoder $\mathbf{Z}$, the non-zero entries of the spectrum-shaped sequences $\mathbf{r}_{f}^{s_{0}}, \mathbf{r}_{f}^{s_{1}}$ are not identical as shown in Fig. 9. This will be shown in asymmetric PAPR and channel estimation performance on port 0 and port 1 , which is not acceptable in any MIMO system.

Using the proposed architecture, it can be shown that the output of the spectrum shaping filter is identical for both ports, i.e.,

$$
\begin{aligned}
r_{f}^{s_{0}}(2 k) & =r_{f}^{s_{1}}(2 k+1) \\
& =r_{f}^{p_{0}}(2 k) w_{f}(2 k)
\end{aligned}
$$

where $\mathbf{r}_{f}^{p_{0}}(k)$ is the $M$ point DFT of $\pi / 2$-BPSK sequence $\tilde{r}_{t}^{p}$. Therefore, the same reference signal is transmitted on each baseband DMRS port, thereby satisfying the specifications provided in [10]. We further show in Section 3 that the estimated channel impulse response will also be the same on both ports, considering identical channel conditions.

The spectrum-shaped DMRS vectors $\mathbf{r}_{f}^{s_{0}}, \mathbf{r}_{f}^{s_{1}}$ are mapped to a set of subcarriers in frequency domain using the matrix $\mathbf{M}_{f}$ as discussed in Section 2.4.1. The resulting output is converted to time domain via inverse-DFT operation similar to the method employed for
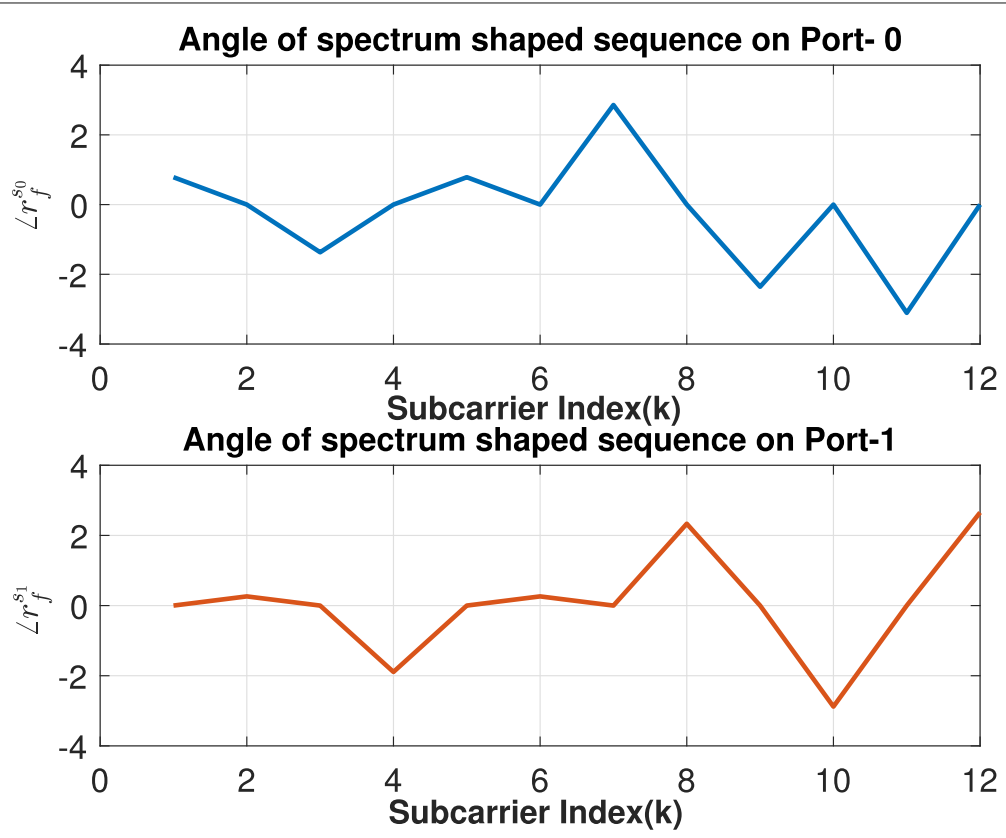

Fig. 9 Angle of $\mathbf{r}_{f}^{s_{0}}, \mathbf{r}_{f}^{s_{1}}$, i.e., the spectrum shaping filter outputs on port 0 and port 1 in the absence of precoder $\mathbf{Z}$ 
data transmission as shown below

$$
\begin{gathered}
\mathbf{s}_{t}^{0}=\mathbf{D}_{N}^{\dagger} \mathbf{M}_{f} \mathbf{r}_{f}^{s_{0}} \\
\mathbf{s}_{t}^{1}=\mathbf{D}_{N}^{\dagger} \mathbf{M}_{f} \mathbf{r}_{f}^{s_{1}} .
\end{gathered}
$$

Using the above, the overall time domain baseband signals $\mathbf{s}_{t}^{0}(t), \mathbf{s}_{t}^{1}(t)$ with an appropriate cyclic prefix are generated as given by equation (5.3.1) in [4].

Note: As mentioned in the introduction section, the latest 5G specifications [9] only support a maximum of 2 spatial users; hence, the transmitter design is discussed in the context of 2 ports. However, the proposed transmitter design can be easily extended to any number of ports. Let "Q" be the total number of spatial users scheduled on a given time frequency resource. The proposed method can be easily extended to generate the "Q" DMRS waveforms with the following minor adjustments.

- The DMRS sequence corresponding to each of "Q" DMRS ports will be of length $\frac{M}{Q}$.

- The $\frac{M}{Q}$ length DMRS is cyclically extended to give a $M$ length vector $\tilde{r}_{t}(n)$ as follows

$$
\tilde{r}_{t}(n)=r_{t}\left(n \bmod \frac{M}{Q}\right), n=0,1, \ldots, M-1 .
$$

- A port-specific precoder $\mathbf{T}_{q}$ is applied on $\tilde{r}_{t}^{q}$, where $\mathbf{T}_{q}$ is a $M \times M$-diagonal matrix with diagonal entries $T_{m m}=e^{i 2 \pi m q / M}$, where $q$ is the port number,

$q=\{0,1,2, \ldots Q-1\}$

$$
\mathbf{r}_{f}^{p_{q}}=\mathbf{D}_{M} \mathbf{T}_{q} \tilde{r}_{t}^{q}
$$

- A port-specific precoder $\mathbf{Z}_{q}$ is applied on the respective spectrum shaping filters of each port to result in a spectrum-shaped DMRS sequence as follows

$$
\mathbf{r}_{f}^{s_{q}}=\left(\mathbf{Z}_{q} \mathbf{w}_{f}\right) \cdot \mathbf{r}_{f}^{p_{q}}
$$

\subsection{Transmission method 2}

In the method 1-based transmitter design, the $\pi / 2$-BPSK data and DMRS sequences are spectrum-shaped in the frequency domain. Further, the DFT-precoded DMRS sequences corresponding to each DMRS port are generated and spectrum-shaped independently; however, some additional processing is required to design the spectrum-shaped sequence for the port 1 compared to port 0 . In the method 2-based design, we propose a low complexity architecture, where spectrum shaping is performed in time domain for both data and DMRS via circular convolution operation. Specifically, a single DMRS sequence is spectrum-shaped in time domain and mapped to both DMRS ports. In contrast to method 1, no additional processing is required to generate the reference sequence for port 1. The architecture for this transmitter design for the data and DMRS is shown in Figs. 10 and 11 , respectively.

\subsubsection{Data waveform design method 2}

Let $\mathbf{x}_{t}$ be the $M$ length data vector to be transmitted from the UE to base station that undergoes a $\pi / 2$-phase rotation through an $M \times M$ diagonal matrix $\mathbf{P}$. Here, $\mathbf{P}$ is the same matrix used in method 1 . This results in an $M$ length data vector $\mathbf{x}_{t}^{p}=\mathbf{P} \mathbf{x}_{t}$ with $\pi / 2$-BPSK symbols. The spectrum shaping of $\frac{\pi}{2}$-BPSK data is performed in time domain through a circular-convolution procedure with zero-padded $\mathbf{w}_{t}$ to produce a spectrum-shaped data 


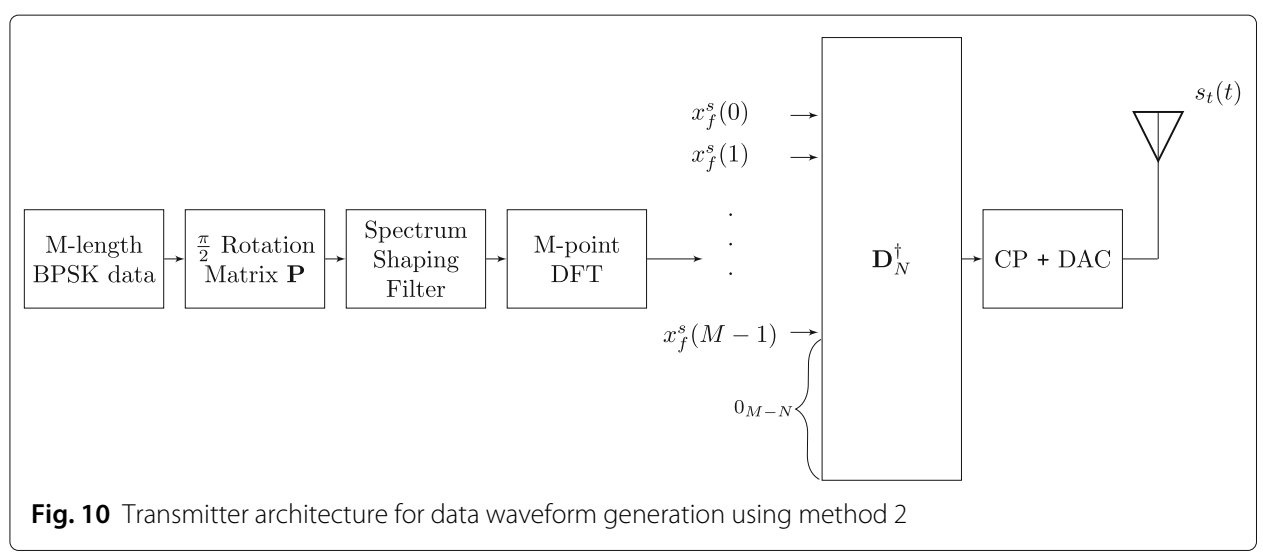

as,

$$
\begin{aligned}
x_{t}^{s}(n)=\sum_{m=0}^{M-1} x_{t}^{p}(n) w_{t}((m+n) \bmod M), \\
\quad m, n=\{0,1,2, \ldots \ldots, M-1\} .
\end{aligned}
$$

The spectrum-shaped data sequence is DFT precoded by means of $M$ point DFT matrix as $\mathbf{x}_{f}^{s}=\mathbf{D}_{M} \mathbf{x}_{t}^{s}$. The DFT precoded spectrum-shaped data vector is mapped to a set of subcarriers in frequency domain via a mapping matrix $\mathbf{M}_{f}$ (described in Section 2.4.1). Finally, this mapped sequence is converted to time domain via inverse-DFT operation as.

$$
\mathbf{s}_{t}=\mathbf{D}_{N}^{\dagger} \mathbf{M}_{f} \mathbf{x}_{f}^{s} .
$$

Using the above, the overall time domain baseband signals $\mathbf{s}_{t}(t)$ with appropriate length cyclic prefix are generated as per equation (5.3.1) in [4].

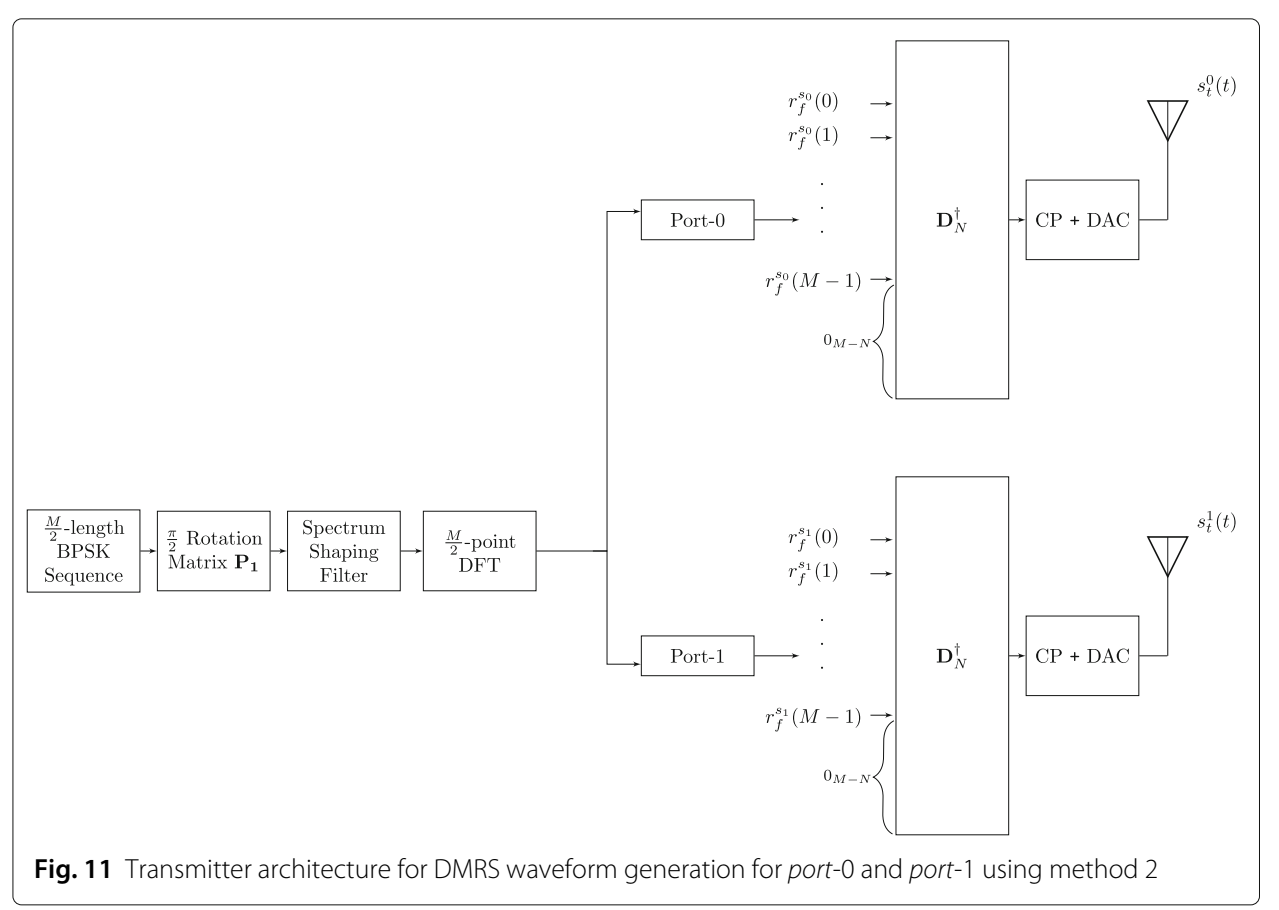




\subsubsection{DMRS waveform design method 2}

Let $\mathbf{r}_{t}$ be the pre-determined $\frac{M}{2}$ length DMRS sequences (as mentioned earlier in method 1 ) with BPSK modulated symbols, which undergo $\pi / 2$-phase rotation through diagonal matrix $\mathbf{P}_{1}$ of sizes $\frac{M}{2} \times \frac{M}{2}$. The diagonal entries of $\mathbf{P}_{1}$ are given by $\left(e^{i(m \bmod 2) \frac{\pi}{2}}\right)$. This results in an $\frac{M}{2}$ length DMRS vector $\mathbf{r}_{t}^{p}=\mathbf{P}_{1} \mathbf{r}_{t}$ with $\pi / 2$-BPSK symbols. The spectrum shaping of the DMRS symbols is performed in time domain through a circularconvolution procedure with zero-padded $\mathbf{w}_{t}$ to produce a spectrum-shaped DMRS sequences as

$$
\begin{array}{r}
r_{t}^{s}(n)=\sum_{m=0}^{\frac{M}{2}-1} r_{t}^{p}(n) w_{t}\left((m+n) \bmod \frac{M}{2}\right), \\
m, n=\left\{0,1,2,3 \ldots \ldots, \frac{M}{2}-1\right\}
\end{array}
$$

The spectrum-shaped DMRS sequence is DFT precoded by means of $\frac{M}{2}$ point DFT matrix as $\mathbf{r}_{f}^{s}=\mathbf{D}_{\frac{M}{2}} \mathbf{r}_{t}^{s}$. The DFT output of DMRS sequence generated above is mapped to port 0 as

$$
\begin{aligned}
r_{f}^{s_{0}}(k) & =r_{f}^{s}\left(\frac{k}{2}\right) & & k \in 0,2,4, \ldots \\
& =0 & & \text { otherwise, }
\end{aligned}
$$

and to port 1 as

$$
\begin{aligned}
r_{f}^{s_{1}}(k) & =r_{f}^{s}\left(\frac{k-1}{2}\right) \quad k \in 1,3,5, \ldots \\
& =0 \quad \text { otherwise. }
\end{aligned}
$$

In the above equations, $\mathbf{r}_{f}^{s_{0}}$ and $\mathbf{r}_{f}^{s_{1}}$ indicate the frequency domain DMRS sequences on port 0 and port 1 respectively. It can be seen that with the proposed architecture the non-zero entries of DMRS sequence are exactly identical for both ports i.e.,

$$
\begin{aligned}
r_{f}^{s_{0}}(2 k) & =r_{f}^{s_{1}}(2 k+1) \\
& =r_{f}^{p}(k) w_{f}(k)
\end{aligned}
$$

where $\mathbf{r}_{f}^{p}(k), \mathbf{w}_{f}(k)$ are the $\frac{M}{2}$-DFT outputs of $\pi / 2$-BPSK DMRS symbol $\mathbf{r}_{t}^{p}$ and filter $\mathbf{w}_{t}$ respectively. The DFT precoded spectrum-shaped data and DMRS vector of each port is mapped to a set of subcarriers in frequency domain via mapping matrix $\mathbf{M}_{f}$ (described in Section 2.4.1). Finally, this mapped sequence is converted to time domain via inverse-DFT operation as

$$
\begin{aligned}
& \mathbf{s}_{t}^{0}=\mathbf{D}_{N}^{\dagger} \mathbf{M}_{f} \mathbf{r}_{f}^{s_{0}} \\
& \mathbf{s}_{t}^{1}=\mathbf{D}_{N}^{\dagger} \mathbf{M}_{f} \mathbf{r}_{f}^{s_{1}} .
\end{aligned}
$$

Using the above, the overall time domain baseband signals for DMRS transmission i.e., $\mathbf{s}_{t}^{0}(t), \mathbf{s}_{t}^{1}(t)$ with appropriate length cyclic prefix are generated as per equation (5.3.1) in [4].

Note: Similar to the transmission method 1, method 2 can also be extended to arbitrary number of scheduled ports " $Q$ " using the following steps: 
- The predefined $\frac{M}{Q}$ length vector $\mathbf{r}_{t}$ with BPSK modulated symbols is $\frac{\pi}{2}$ rotated using an $\frac{M}{Q} \times \frac{M}{Q}$ diagonal matrix $\mathbf{P}_{q}$ as,

$$
\mathbf{r}_{t}^{p}=P_{q} \mathbf{r}_{t} .
$$

where the diagonal entries of $\mathbf{P}_{q}$ are given by $e^{(i(\bmod 2)) \frac{\pi}{2}}$.

- The time domain spectrum shaping filter $\mathbf{w}_{t}$ is used to perform time domain spectrum shaping of $\mathbf{r}_{t}^{p}$ as follows:

$$
\mathbf{r}_{t}^{s}=\sum_{n=0}^{\frac{M}{Q}-1} \mathbf{r}_{t}^{p} \mathbf{w}_{t}(n+m) \bmod \frac{M}{Q} .
$$

- Matrix $D_{\frac{M}{Q}}$ is used to perform DFT precoding as

$$
\mathbf{r}_{f}^{s}=\mathbf{D}_{\frac{M}{Q}} \mathbf{r}_{t}^{s}
$$

- Port-specific mapping for an arbitrary port $q$ is performed as

$$
\begin{aligned}
\mathbf{r}_{f}^{p}(k)=\mathbf{r}_{f}^{s}\left(\frac{k-q}{2}\right) & \\
& \\
& \text { where, } p=\{0,1,2, \ldots . Q-1\} \\
& k=\{p, p+Q, p+2 Q \ldots . . .\}
\end{aligned}
$$

\subsection{Summary of the transmission methods}

We presented two transmission methods for the data DMRS waveform generation. Specifically, in method 1, the processing happens in frequency domain, while in method 2 , the processing happens in the time domain via the circular-convolution operation. In the frequency domain method, the spectrum shaping is performed on the $M$ length sequence. Hence, the spectrum shaping filters must be defined for length $M$; an additional care must be taken to design this spectrum-shaped sequence for the port 1 vs. port 0 , whereas, in the time domain method, the spectrum shaping is performed in the time domain via the circular convolution method. Note that at this stage, the length of the sequence is $M / 2$. Further, the length of the filter is a maximum of 3 taps. Therefore, circular convolution must be performed between a length- 3 and length $\mathrm{M} / 2$ sequences appropriately. In contrast to frequency domain method, no care needs to be taken to generate the reference sequence for port 0 and port 1 in time domain case as the same output sequence can be mapped to both ports.

The following DFT properties are invoked to establish equivalence between transmission method 1 and transmission method 2. For any arbitrary sequence with length less than or equal to $M / 2$, the even coefficients of its $M$ point DFT are identical to its $\mathrm{M} / 2$-point DFT coefficients.

$$
r_{f, M}^{p_{0}}(2 k)=r_{f, \frac{M}{2}}^{p_{0}}(k) \quad, \quad k=\left\{0,1, \ldots . \frac{M}{2}-1\right\}
$$

where $\mathbf{r}_{f, M}^{p_{0}}, \mathbf{r}_{f, \frac{M}{2}}^{p_{0}}$ are $M$ point and $\frac{M}{2}$ point DFT outputs of $\mathbf{r}_{t}^{p}$ respectively.

Using (17), we can rewrite (10) as

$$
\begin{aligned}
r_{f}^{s_{0}}(2 k) & =r_{f}^{s_{1}}(2 k+1) \\
& =r_{f, \frac{M}{2}}^{p_{0}}(k) w_{f, \frac{M}{2}}(k),
\end{aligned}
$$


which is exactly identical to (15). Since input to IDFT is identical for both methods, we conclude that the inverse DFT outputs of port 0 and port 1, i.e., $\mathbf{r}_{f}^{s_{0}}, \mathbf{r}_{f}^{s_{1}}$ and the subsequent baseband signals generated through method 1 will be identical to that of generated using method 2. Using an example, we show in the Appendix that the channel estimation performance when these different transmitter methods are used will remain the same.

\section{Receiver design}

In this section, the receiver architecture to decode the received $\pi / 2$-BPSK data symbols is discussed. The receiver procedure is common for both transmission methods (explained in Section 2). The receiver architecture is shown in Fig. 12.

The receiver front end operations such as sampling, synchronization, CP removal, and FFT are similar to a conventional DFT-s-OFDM-based system. Further, the ISI introduced by the propagation channel is assumed to be less than that of the CP length. Therefore, after CP removal and FFT, the data and DMRS signals on $k$ th subcarrier can be represented as (without loss of generality, we consider only the initial M subcarriers of the DFT output, i.e., $k \in[0, M-1])$

$$
\begin{aligned}
y_{d}(k) & =x_{f}^{s_{0}}(k) h_{f, \mathrm{data}}^{0}(k)+x_{f}^{s_{1}}(k) h_{f, \mathrm{data}}^{1}(k)+v(k) \\
y_{\mathrm{DMRS}}^{0}(k) & =r_{f}^{s_{0}}(k) h_{f, \mathrm{DMRS}}^{0}(k)+v_{0}(k) \\
y_{\mathrm{DMRS}}^{1}(k) & =r_{f}^{s_{1}}(k) h_{f, \mathrm{DMRS}}^{1}(k)+v_{1}(k) .
\end{aligned}
$$

Above, $\mathbf{y}_{d}$ corresponds to the received data vector with data symbols from both ports. $\mathbf{y}_{\mathrm{DMRS}}^{0}, \mathbf{y}_{\mathrm{DMRS}}^{1}$ corresponds to the received DMRS vectors on port 0 and port 1 respectively. $\mathbf{h}_{f, \text { DMRS }}^{0}=\mathbf{D}_{M} \mathbf{h}_{t}^{0}$ and $\mathbf{h}_{f, \text { DMRS }}^{1}=\mathbf{D}_{M} \mathbf{h}_{t}^{1}$ correspond to frequency response of CIR on port $0\left(\mathbf{h}_{t}^{0}\right)$ and port $1\left(\mathbf{h}_{t}^{1}\right)$ respectively, $\mathbf{x}_{f}^{s_{0}}$ and $\mathbf{x}_{f}^{s_{1}}$ are the transmitted data sequences on port 0 and port 1 respectively, and $\mathbf{r}_{f}^{s_{0}}$ and $\mathbf{r}_{f}^{s_{1}}$ are the transmitted DMRS sequences on port 0 and port 1 respectively. The noise vectors $\mathbf{v}, \mathbf{v}_{0}$, and $\mathbf{v}_{1}$ are i.i.d. complex Gaussian random variables with zero-mean and co-variance $\sigma^{2} \mathbf{I}$ where $\mathbf{I}$ is an identity matrix and $\sigma^{2}$ is a constant indicating the variance of each noise sample.

In practice, for low to medium user speeds, the time variations of the multipath channel across consecutive OFDM symbols will be minimal and hence we consider that the channel on the data and DMRS symbol will be same, i.e.,

$$
\begin{aligned}
h_{f, \mathrm{data}}^{0}(k) & =h_{f, \mathrm{DMRS}}^{0}(k) \\
h_{f, \mathrm{data}}^{1}(k) & =h_{f, \mathrm{DMRS}}^{1}(k) .
\end{aligned}
$$

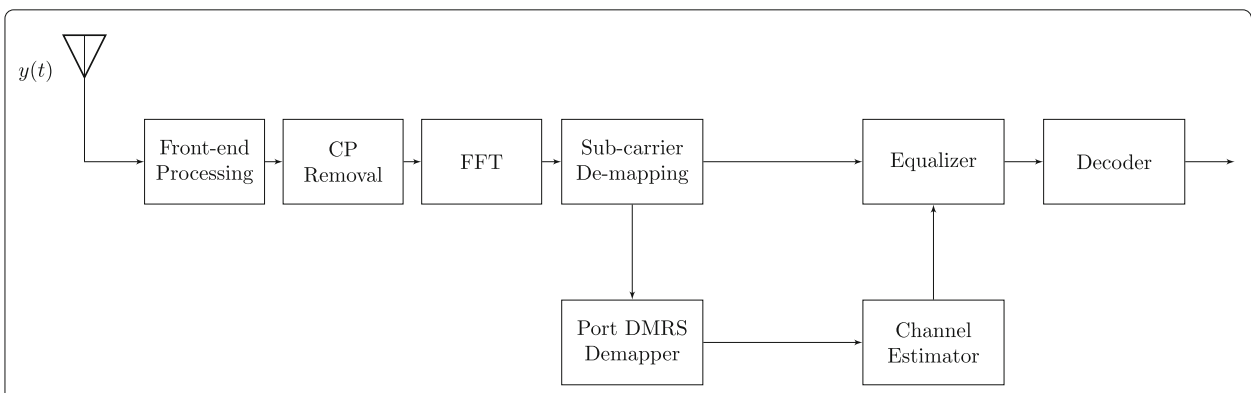

Fig. 12 Base station receiver architecture for each receiver antenna 


\subsection{Channel estimation}

As mentioned in the Section 2.2, the channel estimator needs to estimate the joint impulse response of the spectrum shaping filter and the wireless channel. In our work, we employ a DFT-based channel estimation technique to estimate the joint channel response for the $M$ allocated subcarriers. A simple least squares-based technique with tone averaging or linear interpolation will not be effective in this case due to the presence of the spectrum shaping filter. Tone averaging or linear interpolation is based on the assumption that the channel is constant across consecutive subcarriers, which does not hold in this case, because the spectrum shaping considerably changes channel across consecutive subcarriers as shown in Fig. 5.

The data vector of length $M$ will be associated with $\frac{M}{2}$ length DMRS vector; since the data is carried on $M$ subcarriers, the channel on all of these $M$ subcarriers must be estimated for coherent demodulation. We show that an $M$ length frequency domain channel vector corresponding to $M$ length data symbol can be perfectly constructed from $\frac{M}{2}$-length DMRS sequence for both the ports.

\subsubsection{Channel estimation on port 0}

As mentioned in the Section 2.4.2, port 0 carries DMRS only on even numbered subcarriers. From the received DMRS symbol, the even numbered subcarriers are extracted and expressed in terms of transmitted $\pi / 2$-BPSK based DMRS sequence as follows

$$
\begin{aligned}
\tilde{y}_{\mathrm{DMRS}}^{0}(k) & =y_{\mathrm{DMRS}}^{0}(2 k), k=0,1, . . \frac{M}{2}-1 \\
& =r_{f}^{s_{0}}(2 k) h_{f, \mathrm{DMRS}}^{0}(2 k)+v_{0}(2 k) \\
& =r_{f}^{p_{0}}(2 k) w_{f}(2 k) h_{f, \mathrm{DMRS}}^{0}(2 k)+v_{0}(2 k)
\end{aligned}
$$

where (20) results from (18), and (21) results from (10). Invoking the equivalence between $M$ point DFT and $\frac{M}{2}$-point DFT (17), the above equation can be represented as

$$
\tilde{\mathbf{y}}_{\mathrm{DMRS}}^{0}=\left[\mathbf{D}_{\frac{M}{2}} \mathbf{r}_{t}^{p}\right]\left[\mathbf{D}_{\frac{M}{2}}\left(\mathbf{w}_{t} \odot \mathbf{h}_{t}^{0}\right)\right]+\mathbf{v}_{0},
$$

where $\odot$ indicates the circular convolution operation; $\mathbf{r}_{t}^{p}$ is defined in Section 2.5.2. As mentioned in the Section 2.2, the length of spectrum shaping filter can be a maximum of 3 taps and the reference sequence design $[14,15]$ ensures that the length of DMRS sequence is always greater than impulse response $\mathbf{h}_{t}^{0}$ of any possible wireless channel. Hence, the following holds

$$
\text { length }\left(\mathbf{w}_{t} \odot \mathbf{h}_{t}^{0}\right)=\max \left(\text { length }\left(\mathbf{w}_{t}\right) \text {, length }\left(\mathbf{h}_{\mathrm{t}}^{0}\right)\right) \leq \frac{M}{2} .
$$

We invoke the following DFT relation to derive the joint impulse response. Any arbitrary time domain sequence $\mathbf{g}_{t}$ with length $\mathrm{L} \leq \frac{\mathrm{M}}{2}$ can be reconstructed with only either even or odd coefficients of its M point DFT.

$$
\begin{gathered}
\mathbf{D}_{M}\left(\mathbf{g}_{t}\right)=\mathbf{g}_{f} \\
\mathbf{D}_{\frac{M}{2}}^{\dagger}\left(g_{f}(2 k)\right)=g_{t}(m) \\
\mathbf{D}_{\frac{M}{2}}^{\dagger}\left(g_{f}(2 k+1)\right)=e^{\frac{i 2 \pi n}{M}} g_{t}(m)
\end{gathered}
$$

We perform channel estimation on $\tilde{\mathbf{y}}_{\mathrm{DMRS}}^{0}$ as follows: we first perform a least squaresbased channel estimation followed with an $\frac{M}{2}$ point IDFT. This gives the joint impulse 
response of filter and the wireless channel as

$$
\mathbf{D}_{\frac{M}{2}}^{\dagger}\left(\frac{\tilde{y}_{\text {DMRS }}^{0}}{\mathbf{D}_{\frac{M}{2}}^{0} \mathbf{r}_{t}^{\prime}}\right)=\underbrace{\mathbf{w}_{t} \odot \mathbf{h}_{t}^{0}}_{\mathbf{h}_{\mathrm{eff}}}+\mathbf{v}_{0} .
$$

Invoking the above remarks on lengths of spectrum shaping filter and channel impulse response, it can be deduced that $\mathbf{h}_{\mathrm{eff}}$ completely captures the joint impulse response of the spectrum shaping filter and the wireless channel.

A denoising time domain filter [16] is then applied to reduce noise in (23). This filter $\mathbf{f}(n)$ is defined as

$$
\begin{aligned}
\mathbf{f}(n) & =1, & & 0 \leq n \leq f_{c}-1, M-f_{c} \leq n \leq 1 \\
& =0, & & \text { otherwise }
\end{aligned}
$$

where $f_{c}$ is the "cutoff" point, which is commonly chosen as the length of the wireless channel length $\left(\mathbf{h}_{\mathrm{t}}^{0}\right)$ if it is known a priori; otherwise, it is set to the cyclic prefix length. This filtering extracts only the useful samples of the CIR by excluding the rest of the possible noise samples. The effective impulse response after de-noising is given as

$$
\hat{h}_{\text {eff }}(n)=h_{\text {eff }}(n) f(n), \quad 0 \leq n \leq M-1 .
$$

Lastly, the time domain filtered samples are transformed via an $M$ point DFT to recover the frequency-domain channel estimates on each subcarrier $k \in\{0,1,2, \ldots . . . M-1\}$ as $\hat{\mathbf{h}}_{\text {eff }}^{f}=\mathbf{D}_{M} \hat{\mathbf{h}}_{\text {eff }}$. These channel estimates can be further used for port-0 data demodulation using well-known techniques.

\subsubsection{Channel estimation on port 1}

As mentioned in the Section 2.4.2, port 1 carries DMRS only on odd numbered subcarriers. From the received DMRS symbol, the odd numbered subcarriers are extracted and expressed in terms of transmitted $\pi / 2$-BPSK based DMRS sequence as follows

$$
\tilde{y}_{\text {DMRS }}^{1}(k)=y_{\text {DMRS }}^{1}(2 k+1), k=\left\{0,1, \ldots, \frac{M}{2}-1\right\} .
$$

Using (19), the above equation can be written as

$$
\tilde{y}_{\mathrm{DMRS}}^{1}(k)=r_{f}^{s_{1}}(2 k+1) h_{f, \mathrm{DMRS}}^{1}(2 k+1)+v_{1}(2 k+1) .
$$

Using (10), (24) can be expressed as

$$
\tilde{\mathbf{y}}_{\text {DMRS }}^{1}=\left[\mathbf{D}_{\frac{M}{2}} \mathbf{r}_{t}^{p}\right]\left[\mathbf{D}_{\frac{M}{2}}\left(\mathbf{w}_{t} \odot \mathbf{h}_{t}^{1}\right)\right]+\mathbf{v}_{1} .
$$

Further processing steps such as the least squares-based channel estimation, de-noising, and transforming the effective impulse response to frequency domain are identical to the procedure followed for channel estimation on port 0. For the case of AWGN channel, i.e.,

$$
h_{f, \mathrm{DMRS}}^{0}(k)=h_{f, \mathrm{DMRS}}^{1}(k)=1, \quad \forall k
$$

the estimated joint impulse response $\mathbf{h}_{\text {eff }}$ on port 0 and port 1 is shown in Fig. 13. It can be noticed that the estimated impulse response is identical for both ports.

\subsection{Equalization and data demodulation}

The estimated channel on port 0 and port 1 will be employed for channel equalization of data streams. Specifically, we construct an MMSE filter employing the channel estimates obtained previously. The MMSE filter is then applied to the received signal samples from 

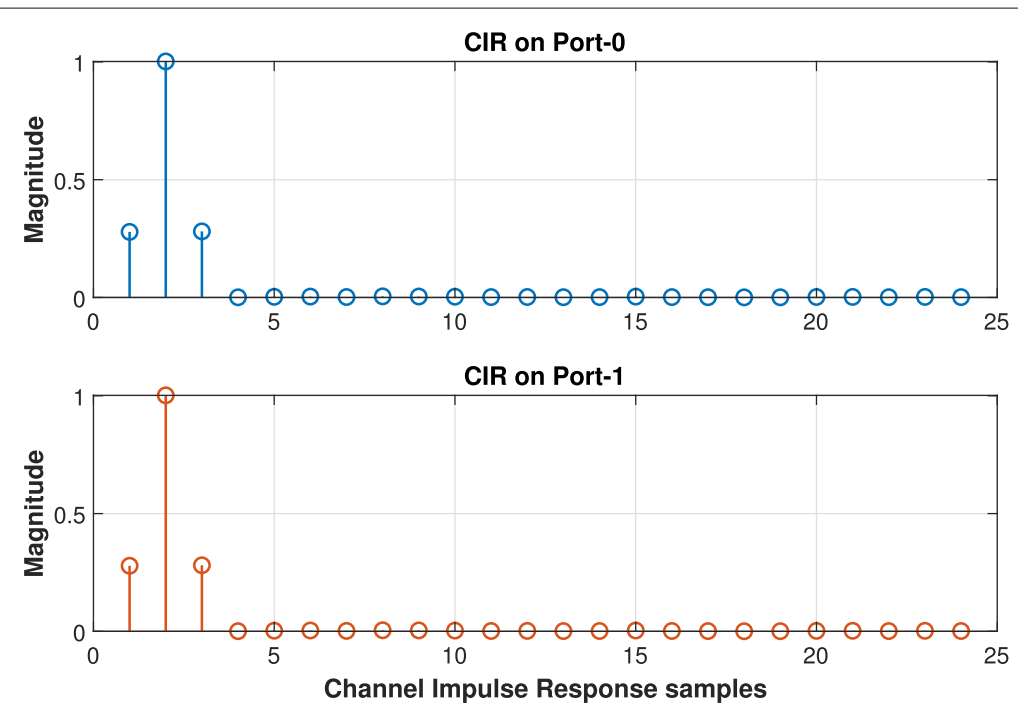

Fig. 13 Magnitude of the estimated channel impulse response on port 0 and port 1

all the receive antennas of the base station to result in equalized data symbols. The equalized data symbols are demodulated to generate soft log-likelihood ratio values, which are subsequently fed to the channel decoder module for subsequent bit-level processing.

\section{Numerical results}

In this section, we present various numerical results that show

- The PAPR comparison between the $\pi / 2$-BPSK-based DMRS sequences and the existing ZC or CGS-based DMRS sequences.

- Link level block error rate (BLER) comparison for the data transmissions employing $\pi / 2$-BPSK-based DMRS sequences and existing ZC or CGS-based DMRS sequences for various sequence lengths and various bandwidth allocations.

- Link throughput vs. SNR comparison for the data transmissions employing $\pi / 2$-BPSK-based DMRS sequences and existing ZC or CGS-based DMRS sequences for various sequence lengths.

- BLER performance for the data transmissions on port 0 and port 1 in the case of MIMO two-stream transmissions.

Unless otherwise mentioned, the simulation assumptions shown in Table 2 are used throughout this paper.

The CCDF of PAPR for ZC sequences and $\pi / 2$-BPSK-based DMRS sequences is shown in Fig. 14. The ZC sequences considered in this case are as defined in [4, Section 5.2.2] with length 96. The PAPR of ZC sequences with and without spectrum shaping is shown in the figure. As can be seen from the figure, the $\mathrm{ZC}$ sequences without spectrum shaping have a PAPR (at the $10^{-3} \mathrm{CDF}$ point) of $2.8 \mathrm{~dB}$ more than the $\pi / 2$-BPSK-based DMRS sequences. When spectrum shaping is applied to the ZC sequences, the PAPR is slightly reduced from that of unfiltered ZC sequences. However, the PAPR of the filtered ZC sequence is still $2.0 \mathrm{~dB}$ larger than the PAPR of the $\pi / 2$-BPSK-based DMRS 
Table 2 Simulation assumptions for BLER comparisons

\begin{tabular}{ll}
\hline Parameter & Value \\
\hline Channel type & PUSCH \\
System bandwidth & $20 \mathrm{MHz}$ \\
Subcarrier spacing & $15 \mathrm{KHz}$ \\
Allocated PRBs & $1-16 \mathrm{PRBS}$ \\
Channel model & TDL-C 300ns \\
Number of UE transmitter antennas & 1 \\
Number of UEs & 1 \\
Number of BS receiver antennas & 2,4 \\
Number of MIMO streams & 1,2 \\
Channel coding & $3 \mathrm{GPP}$ NR LDPC \\
Equalizer & MMSE \\
\hline
\end{tabular}

sequences with the same spectrum shaping. Moreover, as we increase the number of allocated subcarriers for data transmission, the PAPR gap between 3GPP ZC sequence and $\pi / 2$-BPSK-based DMRS sequence increases even further.

The CCDF of QPSK CGS-based DMRS and $\pi / 2$-BPSK-based DMRS for smaller lengths $(M=12)$ is shown in Fig. 15. As discussed in Section 2, for smaller lengths $(M<30)$, 3GPP employs computer-generated sequences (CGS) as DMRS. It can be seen from the figure that the PAPR of the spectrum-shaped CGS sequences is almost $1.2 \mathrm{~dB}$ larger than the PAPR of the $\pi / 2$-BPSK sequences. Moreover, it can also be noticed that for CGS, the PAPR is further increased with filtering, because for shorter lengths, the frequency variations of the spectrum-shaping filter across subcarriers are quite rapid. The IFFT size of the OFDM modulator $(N)$ will be very much larger than sequence length $(M)$; hence, there will be many unused subcarriers at both ends of the sequence and results in the

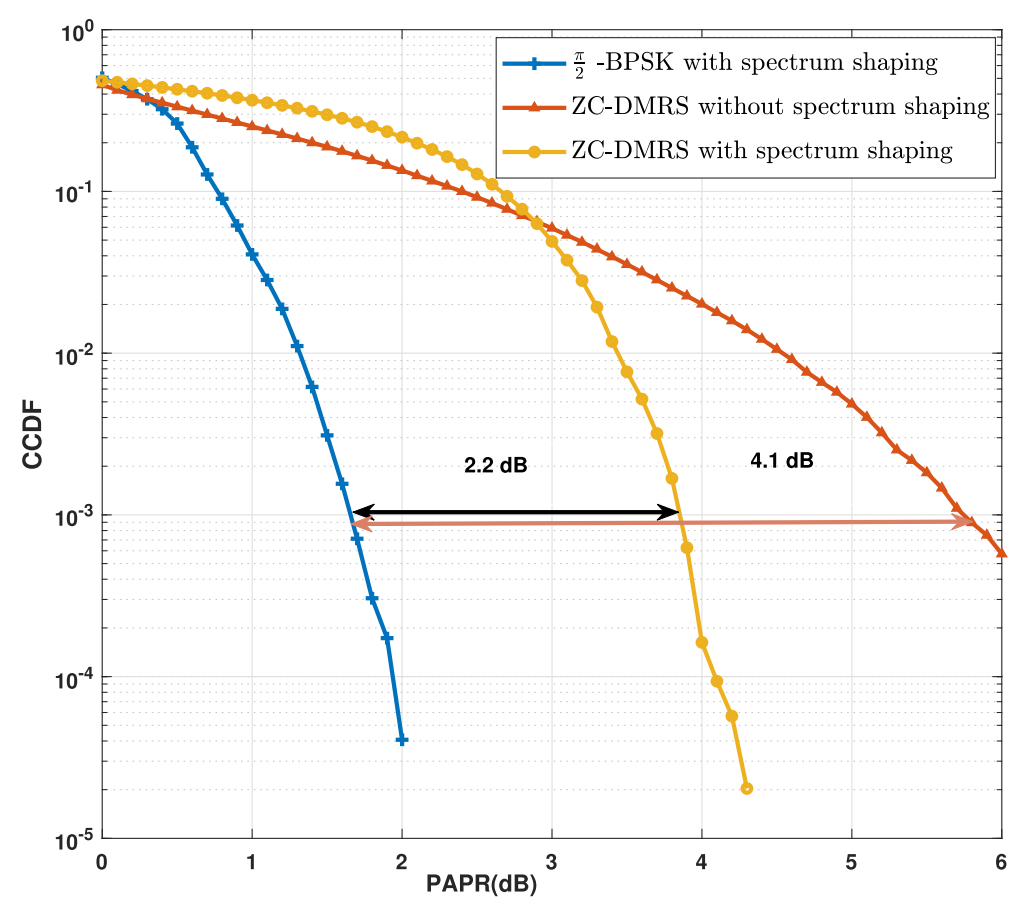

Fig. 14 PAPR of length $96 \mathrm{ZC}$ and $\pi / 2-B P S K$ DMRS sequences 


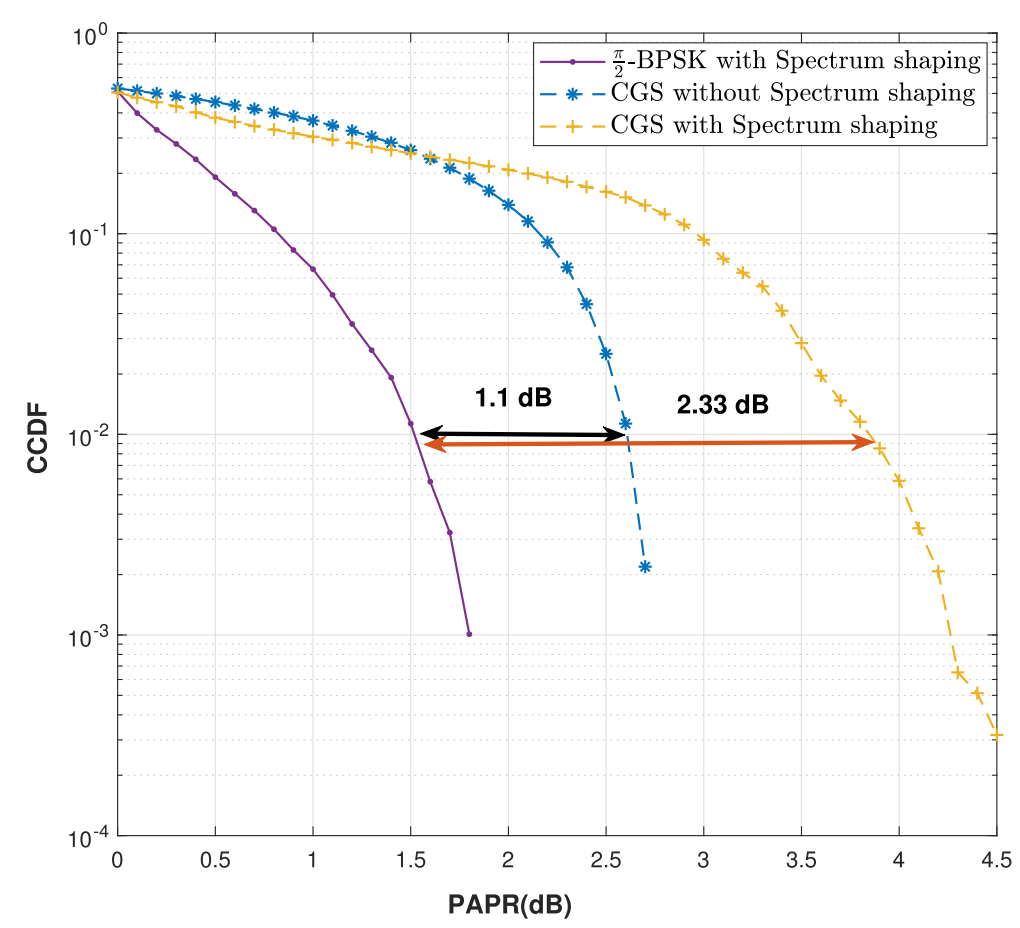

Fig. 15 PAPR of length-12 3GPP CGS and $\pi$ /2-BPSK DMRS sequences

spectrum-shaped sequence effectively being oversampled in the time domain. This significantly affects the spectrum flatness of the CGS. The OFDM outputs of CGS sequences with and without spectrum shaping are shown in Fig. 16. The large amplitude variations in OFDM output of spectrum-shaped CGS sequences can be seen from the figure. Hence, the results shown in Figs. 14, 15 conclude that the $\pi / 2$-BPSK sequences designed in [13] are far superior compared to the existing sequences in improving the cell coverage.

The block error rate performance for a single stream PUSCH transmission is shown in Fig. 17. Here, DMRS is transmitted on port 0 . Note that $\mathrm{ZC}$ sequences are used for comparing the BLER performance because these sequences have a flat frequency spectrum. The frequency flatness ensures unbiased channel estimation across all the allocated subcarriers, which results in the best channel estimation performance. Hence, the goal for the newly designed sequences is to ensure that they match the performance of these $\mathrm{ZC}$ sequences. In this figure, the results are shown for the cases when the base station receiver employs 2 and 4 receive antennas. From Fig. 18, it can be seen that irrespective of the number of receive antennas, the link level performance of $\pi / 2$-BPSK DMRS sequence is equivalent to that of existing CGS based DMRS sequences, although the newly designed sequences are not frequency flat .

The throughput vs. SNR comparisons are shown in Figs. 19 and 20 for the DMRS lengths 96 and 24 respectively. The link throughput is computed by invoking the HybridARQ protocol (HARQ) with a maximum of 4 retransmissions. The throughput shown is the percentage of transport blocks that got decoded correctly, i.e., throughput $=\frac{B_{D}}{B} \times 100$. Here " $B_{D}$ " is the number of transport blocks that got decoded correctly, and " $B$ " is the total number of transport blocks transmitted. It can be seen from the Figs. 19 and 20 that the throughput performance of $\pi / 2$-BPSK DMRS is equivalent to that of existing $\mathrm{ZC}$ and 


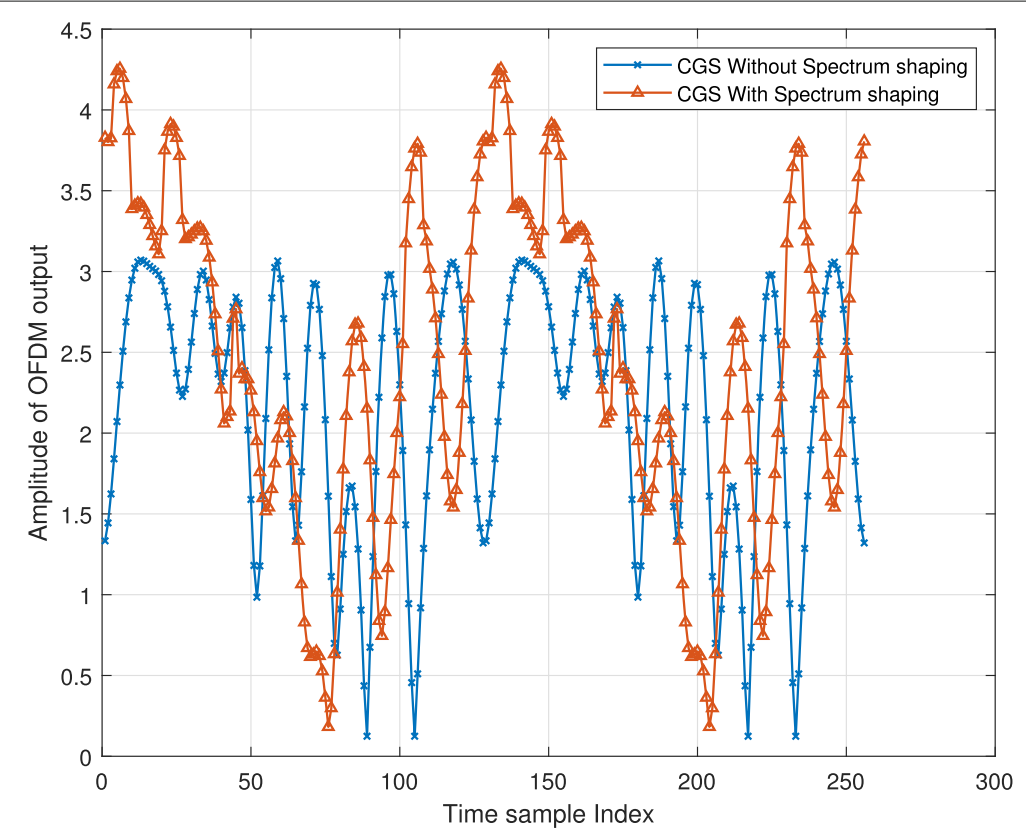

Fig. 16 Magnitude response of CGS sequences with and without spectral shaping

CGS based sequences. From the BLER and throughput analysis, it can be deduced that the $\pi / 2$-BPSK DMRS sequences do not cause any performance degradation but minimizes the PAPR.

We next consider the performance of the proposed transmitter designs for the case of two MIMO streams transmission where DMRS is transmitted on both port 0 and port 1 .

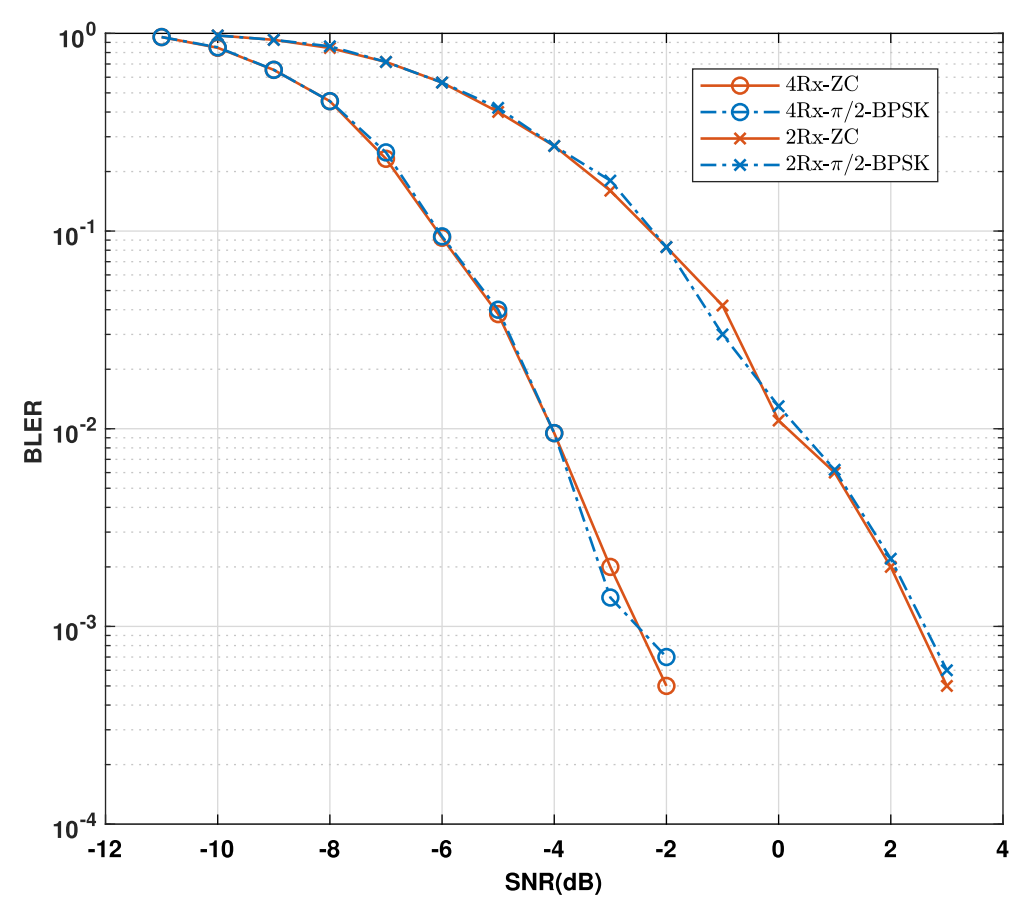

Fig. 17 BLER comparison of length-96 ZC and $\pi / 2-B P S K$ DMRS sequences 


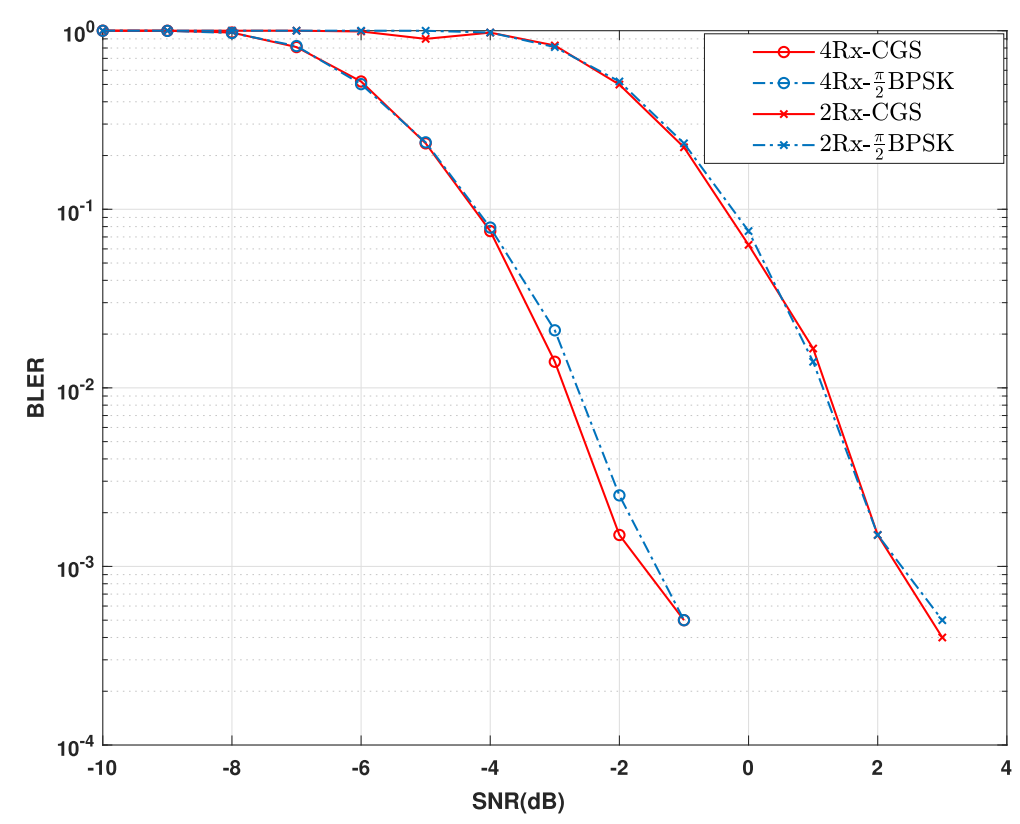

Fig. 18 BLER comparison of length-24 CGS and $\pi / 2$-BPSK DMRS sequences

Firstly, we show the drawbacks of the existing design in 3GPP in Figs. 21 and 22. It can be seen that when the 3GPP transceiver is used, there is a clear difference in the performance both in terms of PAPR and BLER across port 0 and port 1 . This is highly undesirable as the data on two different ports will behave differently. This problem is addressed using the proposed transceiver design, as claimed earlier. We next show that it is indeed the case.

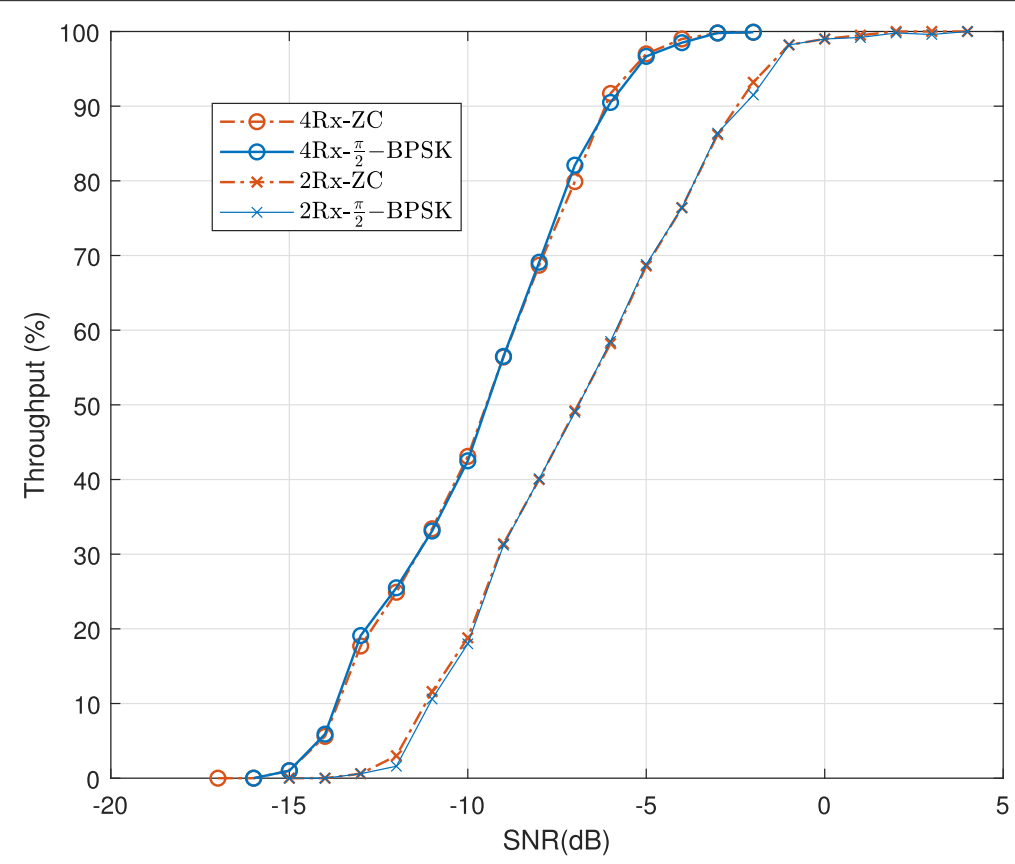

Fig. 19 Throughput comparison for the length-96 DMRS sequences 


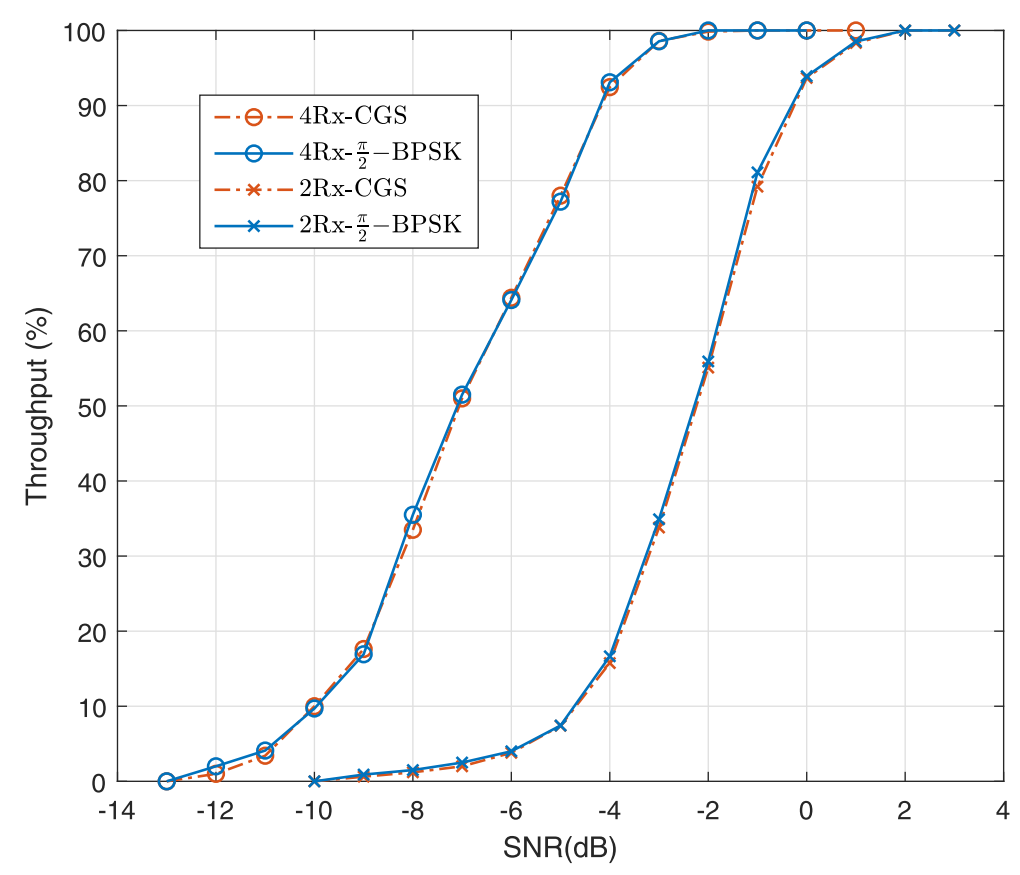

Fig. 20 Throughput comparison for the length-24 DMRS sequences

In Figs. 21 and 23, we show the PAPR and BLER performance for the two MIMO streams transmission setting where DMRS is transmitted on both port 0 and port 1 using our proposed method 1 transceiver design. It can be seen that both PAPR, as well as BLER, are identical for both ports confirming that the proposed transmitter design produces identical DMRS sequences on both ports.

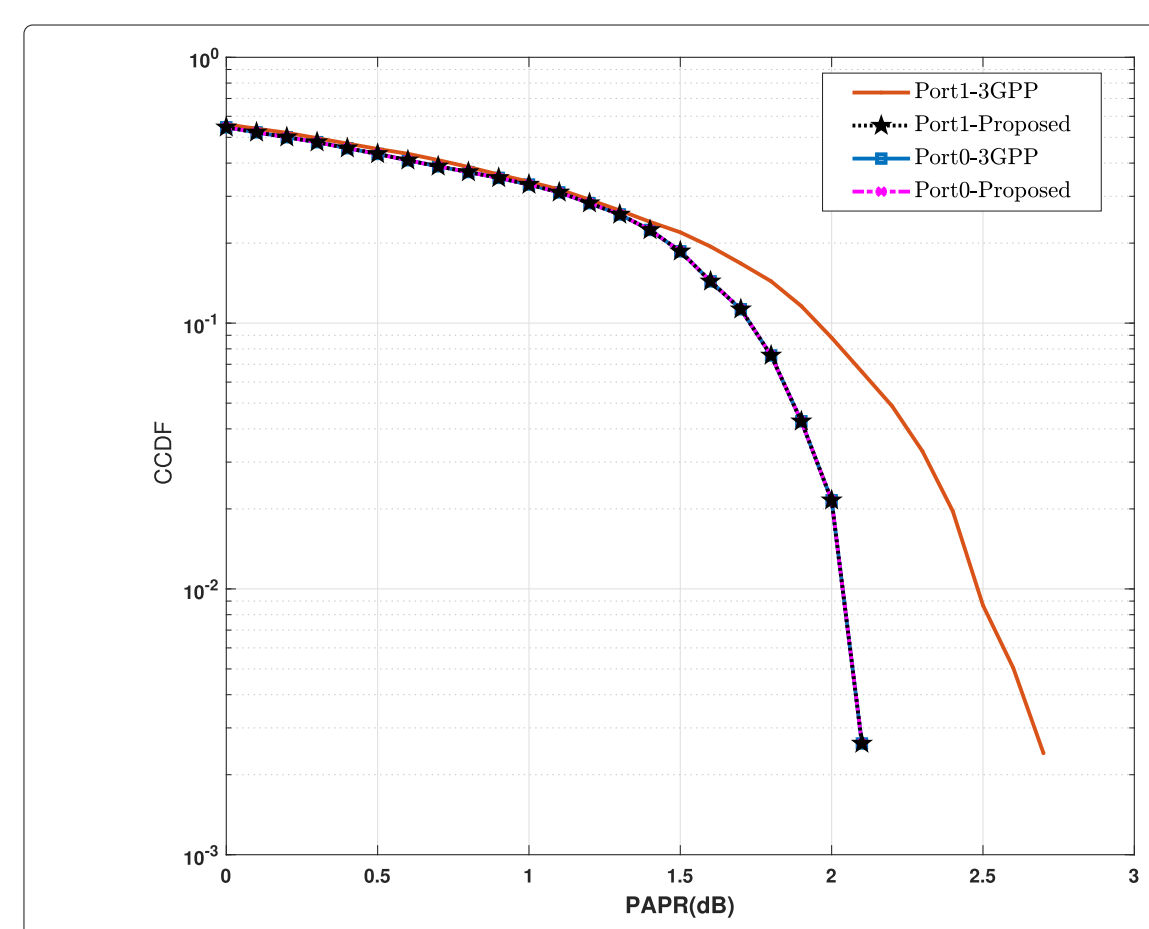

Fig. 21 PAPR comparison of length-6 DMRS sequences on port 0 and port 1 with proposed and 3GPP transmitter designs 


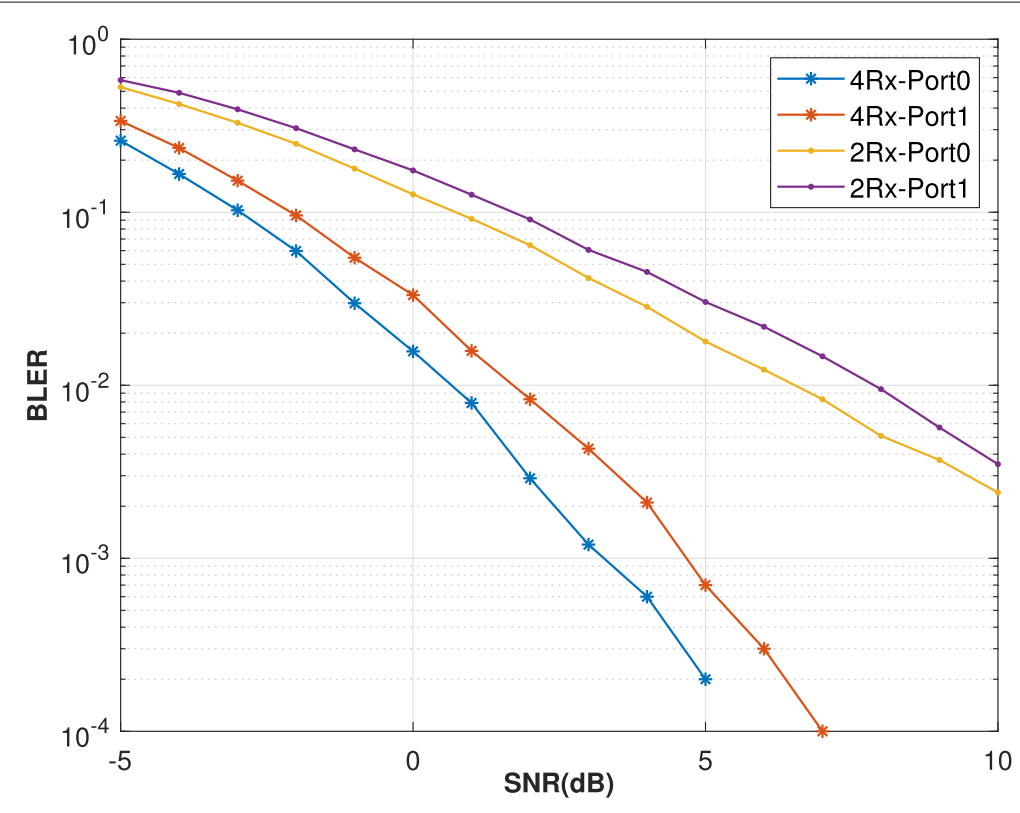

Fig. 22 BLER comparison of length-6 DMRS sequences on port 0 and port 1 with 3GPP transmitter design

In Fig. 24, PAPR of the DMRS sequences on port 0 and port 1 generated by method 1 and method 2 is shown. It can be seen that PAPR is the same for both port 0 and port 1 in both methods confirming that the proposed transmitter designs are equivalent. The same is the case with BLER performance as well. Therefore, the proposed methods 1 and 2 have shown to be equivalent to both analytically and numerically.

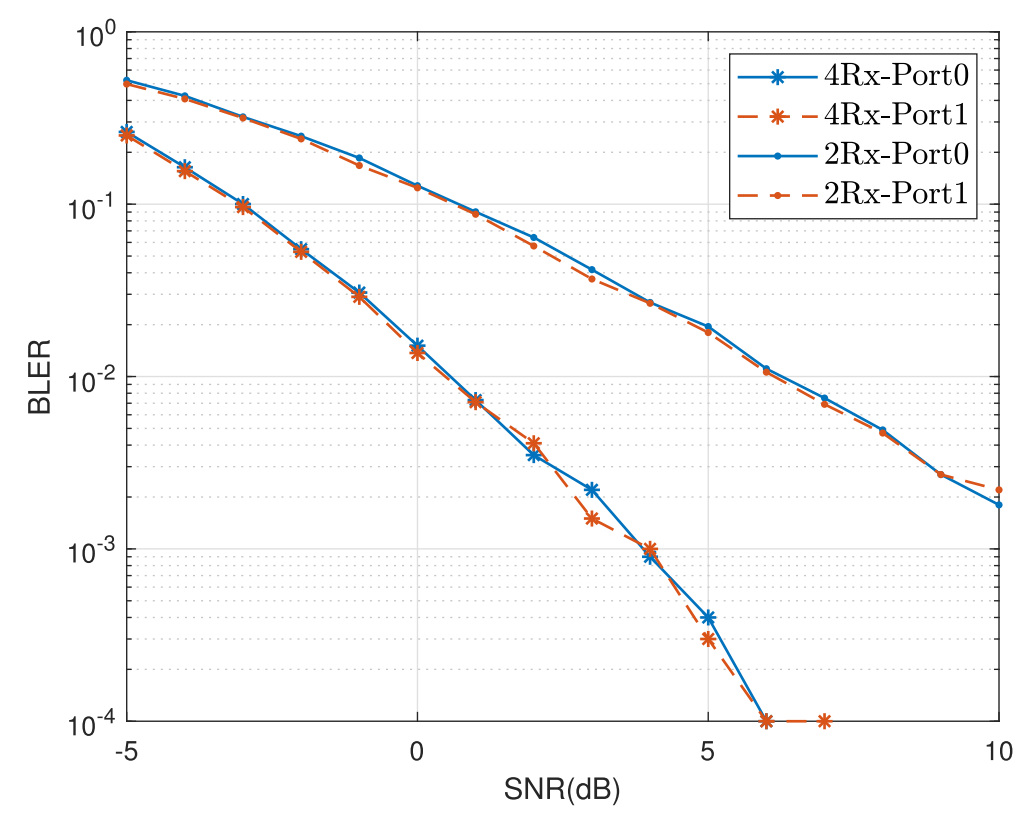

Fig. 23 BLER comparison of length-6 DMRS sequences on port 0 and port 1 with proposed transmitter design 


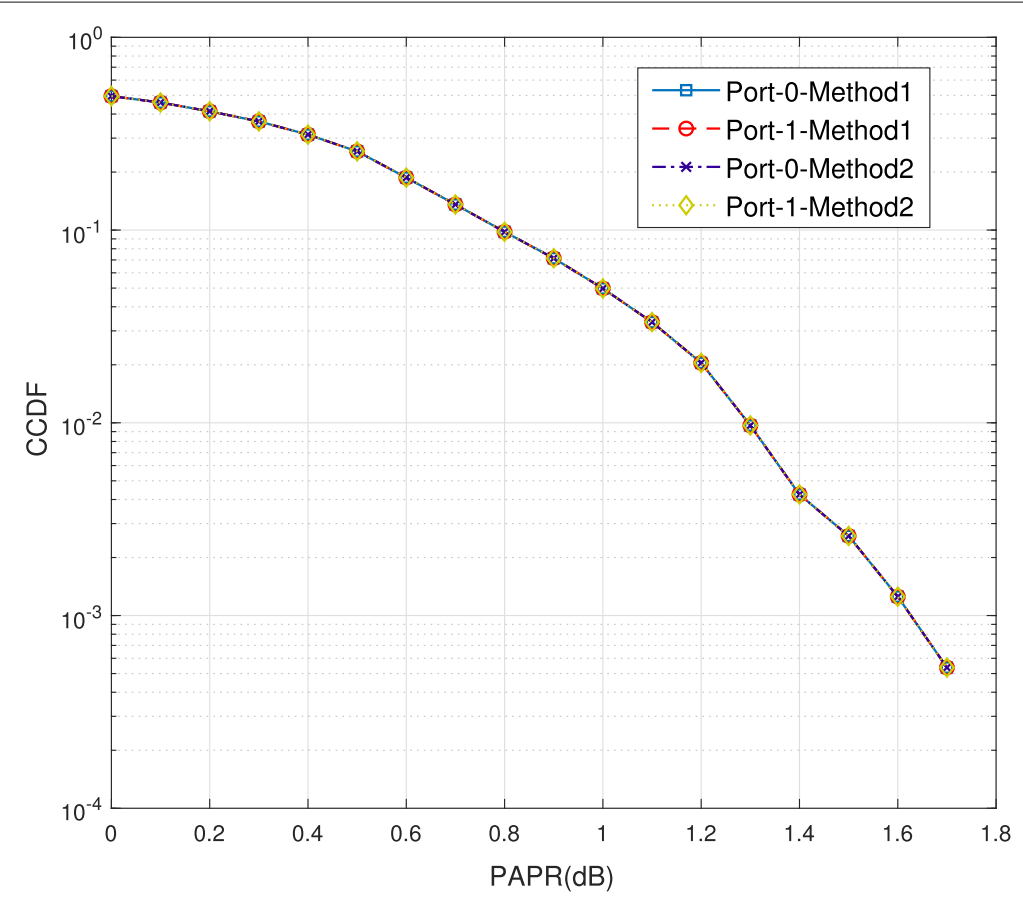

Fig. 24 PAPR comparison of length-12 DMRS sequences on port 0 and port 1 with method 1 and method 2 transmitter designs respectively

\section{Conclusion}

In this paper, a low PAPR reference signal transceiver design for 3GPP 5G NR $\pi / 2$-BPSKbased uplink transmissions is proposed. The PAPR of the reference signal is significantly minimized compared to the current design of Rel-15 5G NR systems using the proposed design. Such a design considerably helps to improve the coverage of the 5G systems. Specifically, we have shown a frequency domain and a time domain transceiver design, both of which are equivalent and result in the same system performance in terms of PAPR and also BLER. We have shown how the proposed design can be extended to the case of a MIMO transmission without causing any discrepancy on different MIMO streams, which is not the case for the current Rel-15 3GPP 5G NR uplink design.

Table 3 DMRS output on port-0, port-1

\begin{tabular}{lll}
\hline Subcarrrier Index & Port 0 & Port 1 \\
\hline 0 & $-0.6223-1.2445 i$ & $0.000+0.000 i$ \\
1 & $0.000+0.000 i$ & $-0.6223-1.2445 i$ \\
2 & $-0.3727-1.3909 i$ & $0.000+0.000 i$ \\
3 & $0.000+0.000 i$ & $-0.3727-1.3909 i$ \\
4 & $2.4728+0.6626 i$ & $0.000+0.000 i$ \\
5 & $0.000+0.000 i$ & $2.4728+0.6626 i$ \\
6 & $4.1412+2.206 i$ & $0.000+0.000 i$ \\
7 & $0.000+0.000 i$ & $4.1412+2.206 i$ \\
8 & $-0.6626-2.4728 i$ & $0.000+0.000 i$ \\
9 & $0.000+0.000 i$ & $-0.6626-2.4728 i$ \\
10 & $1.3909+0.3737 i$ & $0.000+0.000 i$ \\
11 & $0.000+0.000 i$ & $1.3909+0.3737 i$ \\
\hline
\end{tabular}


Table 4 Effective CIR on port-0, port-1

\begin{tabular}{llc}
\hline Time index & Port 0 & Port 1 \\
\hline 0 & -0.28 & -0.28 \\
1 & 1 & 1 \\
2 & -0.28 & -0.28 \\
\hline
\end{tabular}

\section{Methods/experimental}

The transmit power for the uplink transmissions is significantly lesser than the downlink transmissions. Hence, to improve the power efficiency of the uplink transmissions and subsequently the cell coverage, it is required that the uplink waveform to have a low peak to average power ratio (PAPR). To support the low PAPR transmissions, a new modulation scheme, namely $\pi / 2$-BPSK, was introduced in the Rel-15 3GPP 5G NR specifications. However, the reference signals employed for data demodulation have higher PAPR than the data signals, which potentially limits the cell coverage. Also, in the case of spatial multiplexing, the reference signal design should ensure that each data stream experiences a similar channel estimation performance. In this contribution, we present two transmitter designs, namely method 1 and method 2, to generate low PAPR data and reference signals. The proposed transmitters generate $\pi / 2$-BPSK-based reference signals with appropriate spectrum shaping and DFT precoding such that the reference signals on each DMRS port have similar PAPR and results in similar channel estimation performance. In method 1, the processing happens in the frequency domain, where an M/2 length binary sequence is cyclically extended and phase-modulated to result in $M$ length $\pi / 2$-BPSK sequence, which is further DFT precoded and spectrum shaped. In method 2, the processing happens in the time domain via the circular-convolution operation. To decode the received $\pi / 2$-BPSK data symbols, we propose a receiver architecture which is common for both the transmission methods. We justify our claims using computer simulations.

\section{Appendix}

In this section, we present the spectrum-shaped DFT outputs of port 0 and port 1 generated using the proposed transmitter design. In Table 4, we present the effective CIR estimated from both ports in a noise-less scenario. We consider a 3-tap spectrum shaping filter with impulse response $w_{t}=[-0.281-0.28]$. For convenience, we consider a flat and identical wireless channel for both DMRS ports, i.e., $\mathbf{h}_{f, \mathrm{DMRS}}^{0}(k)=\mathbf{h}_{f, \mathrm{DMRS}}^{1}(k)=$ $1 \forall k \in[0, M-1]$. Let $\mathbf{r}_{t}=[111011]$ be a 6-length low PAPR DMRS sequence corresponding to length-12 data allocation. This sequence is passed through the transmitter design as shown in Figs. 7 and 8 or 11 corresponding to the method 1 or method 2 transmitter designs. The resulting output is shown in Table 3. As proved earlier, it can be seen from this table that the non-zero entries of the DMRS sequences on both DMRS ports are the same.

For this setting, the channel can be estimated perfectly on both baseband antenna ports, as shown in Table 4, thereby allowing for correct data demodulation.

\section{Abbreviations}

PAPR: Peak-to-average power ratio; 3GPP: 3rd Generation Partnership Project; NR: New radio; BPSK: Binary phase-shift keying; QAM: Quadrature amplitude modulation; DFT-s-OFDM: Discrete Fourier transform spread orthogonal frequency division multiplexing; DMRS: De-modulation reference signals; TDL: Tapped delay line; LDPC: Low-density parity-check; MMSE: Minimum mean squared error; FFT: Fast Fourier transform; IDFT: Inverse discrete Fourier transform; DFT: Discrete Fourier transform; BLER: Block error rate; CCDF: Complementary cumulative distribution function; PRB: Physical resource block; MIMO: Multiple input-multiple output; CIR: Channel impulse response. 


\section{Authors' contributions}

The transceiver designs proposed in the paper are mainly developed and analyzed by M Sibgath Ali Khan and Koteswara Rao, where M Sibgath Ali Khan is also responsible for generating all the required performance plots and writing of the manuscript till the final submission. Koteswara Rao and Saidhiraj Amuru have extensively reviewed and edited the manuscript to improve the quality of the manuscript. Kiran Kuchi being the supervisor of M Sibgath Ali Khan monitored the whole work from transceiver design till the final submission. All authors read and approved the final manuscript.

\section{Funding}

This work is supported by the Department of Telecommunications(DOT), grant funded by the Ministry of Communications, Government of India (No. 4-23/5G Test Bed /2017-NT dt. 22.03.18 Indigenous 5G Test Bed (Building an end to end $5 \mathrm{G}$ Test Bed)).

\section{Competing interests}

The authors declare that they have no competing interests.

Received: 6 December 2019 Accepted: 14 August 2020

Published online: 22 September 2020

\section{References}

1. 3GPP, TS 38.101 V 15.5.0: User equipment transmission and reception (2019)

2. IITH, et al, R1-1701180: Comparison of pi/2 BPSK with and without frequency domain pulse shaping: results with PA model. (3GPP TSG-RAN WG1 Ad-Hoc NR Meeting, Spokane, 2017)

3. K. Kuchi, Partial response DFT-precoded-OFDM modulation. IEEE Trans. Emerg. Telecommun. Tech. 23(7), 632-645 (2012)

4. 3GPP, TS 38.211 V 15.5.0: Physical channels and modulation (2019)

5. 3GPP, TS 38.213 V 15.5.0: Physical layer Procedures for data (2019)

6. 3GPP, Enhancements for MIMO for NR. (TSG RAN Plenary Meeting 80, La Jolla, 2018)

7. M. S. A. Khan, K. Rao, S. Amuru, K. Kuchi, in The Proceedings of International Conference on COMmunication Systems \& NETworkS (COMSNETS 2020), Low PAPR DMRS sequence design for 5G-NR uplink, (Bengaluru, India, 2020), pp. 207-212

8. Chairman Notes, 3GPP TSG RAN WG1 Meeting Ad-Hoc Meeting 1901. Taipei, Taiwan (2019)

9. 3GPP, TS 38.211 V 16.0.0: Physical channels and modulation (2019)

10. 3GPP, Chairman Notes, TSG RAN WG1 Meeting 96bis, (Xian, 2019)

11. J. Kim, Y. H. Yun, C. Kim, J. H. Cho, Minimization of PAPR for DFT-spread OFDM with BPSK symbols. IEEE Trans. Veh. Technol. 67(12), 11746-11758 (2018)

12. R. Negi, J. Cioffi, Pilot tone selection for channel estimation in a mobile OFDM system. IEEE Trans. Consum. Electron. 44(3), 1122-1128 (1998)

13. Qualcomm, Joint proposal on length-12, length-18, and length-24 CG sequences for pi/2 BPSK. 3GPP TSG RAN WG1 Ad-Hoc Meeting \#1901, R1-1901362, Taipei, Taiwan (2019)

14. 3GPP, Study on channel model for frequencies from 0.5 to $100 \mathrm{GHz}$. 3rd Generation Partnership Project (3GPP), TR 38.901 V $14.3 .0(2017)$

15. P. Hoeher, Kaiser S., P. Robertson, in Multi-carrier spread-spectrum, chapter IV. ed. by K. Fazel, G. P. Fettweis, Pilot-symbol-aided channel estimation in time and frequency (Kluwer Academic Publishers, Dordrecht, The Netherlands, 1997), pp. 169-178

16. Y. Kang, K. Kim, H. Park, Efficient DFT-based channel estimation for OFDM systems on multipath channels. IET Commun. 1(2), 197-202 (2007)

\section{Publisher's Note}

Springer Nature remains neutral with regard to jurisdictional claims in published maps and institutional affiliations.

\section{Submit your manuscript to a SpringerOpen ${ }^{\circ}$ journal and benefit from:}

- Convenient online submission

- Rigorous peer review

Open access: articles freely available online

High visibility within the field

- Retaining the copyright to your article

Submit your next manuscript at $\boldsymbol{\triangleright}$ springeropen.com 